Article

\title{
Optimal Operational Scheduling of Reconfigurable Multi-Microgrids Considering Energy Storage Systems
}

\author{
Saeid Esmaeili ${ }^{1}$, Amjad Anvari-Moghaddam ${ }^{2, *}$ and Shahram Jadid ${ }^{1}$ \\ 1 Department of Electrical Engineering, Iran University of Science and Technology, 16846-13114 Tehran, Iran; \\ s_esmaeili@elec.iust.ac.ir (S.E.); jadid@iust.ac.ir (S.J.) \\ 2 Department of Energy Technology, Aalborg University, 9220 Aalborg East, Denmark \\ * Correspondence: aam@et.aau.dk; Tel.: +45-9356-2062
}

Received: 20 March 2019; Accepted: 4 May 2019; Published: 9 May 2019

check for updates

\begin{abstract}
This paper proposes an optimal operational scheduling of a reconfigurable multi-microgrid (MG) distribution system complemented by demand response programs and Energy Storage Systems (ESSs) in an uncertain environment. Since there is a set of competing players with inherently conflicting objectives in the system under study such as the Distribution System Operator (DSO) and MG owners, a one-leader multi-follower-type bi-level optimization model is proposed. In this framework, the upper-level player as a leader minimizes the total cost from DSO's point of view, while the lower-level players as multi-followers maximize the profit of MG owners. Since the resulting model is a non-linear bi-level optimization problem, it is transformed into a single-level mixed-integer second-order cone programming problem through Karush-Kuhn-Tucker conditions. The satisfactory performance of the proposed model is investigated on a real-test system under different scenarios and working conditions.
\end{abstract}

Keywords: bi-level problem; distribution system; energy storage system; hourly reconfiguration; multi-microgrids; operational scheduling

\section{Introduction}

Recently, due to the energy crisis and environmental deteriorations, the penetration level of Distributed Energy Resources (DERs) including Energy Storage Systems (ESSs), dispatchable and renewable energy sources in distribution sector has been increased significantly. This growing tendency has led to defining the Microgrid (MG) concept as a promising solution to the optimal use of energy resources [1]. Moreover, it mitigates the monitoring and management burden for the Distribution System Operator (DSO) and increases the operational flexibility of the system in the modified decision-making environment of sustainable energy systems [2]. Specifically, a MG is a cluster of loads and DERs which can be operated in both grid-connected and islanded modes [3]. Different aspects related to the application of MGs in distribution systems regarding the analysis and optimization of energy processes including optimal planning [4], operational scheduling [5] and system reliability [6] were thoroughly investigated in the literature. In Reference [7], DSO, who has the responsibility of operating the distribution system in an optimal fashion, determines day-ahead scheduling of DERs using particle swarm optimization (PSO) algorithm. In Reference [8], a two-phase optimal short-term operational scheduling of utility-owned DERs in an active distribution system has been proposed. In the first phase, to determine the amount of purchased power from the wholesale market and the set-points of DERs, a day-ahead energy acquisition model has been developed, where in the second-phase hourly network reconfiguration has been implemented. A day-ahead scheduling 
method in distribution system considering Demand Response (DR) programs has been proposed in Reference [9]. To minimize the total operating cost and pollutants emission (as defined objectives), the augmented $\varepsilon$-constraint method is utilized by the DSO.

In References [6-9] the decision authority is only devoted to the DSO to schedule DERs through a Distribution Management System (DMS) framework. However, with MG implementation, DSO and MG Owners (MGOs) may have conflicting objectives in making decisions. Thus, it is required to establish a comprehensive decision-making model to meet the conflicting objectives desired by different stakeholders. In Reference [10], the day-ahead scheduling of DSO and MGs as the self-governing systems was modelled by the bi-level system of systems framework. At the first step, the problem is solved for MGs and then it is solved for the DSO in an iterative process. Although an appropriate framework has been proposed, the optimization problem is not modelled simultaneously for DSO and MGs. Authors of [11] propose a bi-level DSO-MGs model for optimal operation of the system. The upper-level optimization problem determines the optimal dispatch of distribution system to achieve minimum power loss and voltage deviation, where the lower-level problem takes minimum MGs' operating cost as the objective. Likewise, to minimize carbon emissions, a game between DSO and MG to obtain operational equilibrium strategies is established in Reference [12]. However, the interactions between the two levels have not been implemented in models presented in References [11,12]. In Reference [13], a bi-level methodology to develop the interaction between electric vehicles and DSO considering load demand and wind power uncertainties has been provided. The main drawback of [13] is that each level (DSO and parking lots) has been modelled as a single bus system without considering the constraints of the network. The operational planning of the multi-MG distribution system with local exchanged power has been proposed in References [14-16]. The optimal scheduling model in Reference [14] is solved using a classical optimization strategy, while metaheuristic algorithms are applied for problem solving in References $[15,16]$. The major drawback of [14-16] is devotion of the decision authority to the MGOs without considering the interactions between DSO and each MG, which is addressed in Reference [17]. Bahramara et al. [18] propose a bi-level optimization algorithm, which models the hierarchical decision-making environment of distribution system, where DSO and multi-MGs optimize their respective objectives in a cooperative manner. The resulting bi-level optimization problem is then transformed into a single-level problem through the Karush-Kuhn-Tucker (KKT) optimality conditions. Likewise, in Reference [19], a multi follower bi-level model for optimal decision making of DSO and multi-MGs is presented. In the upper-level problem, the total operation cost is minimized, while in the lower-level problem the profit of each MG is maximized. In Reference [20], a Stackelberg game between a utility company and multiple users aimed at balancing supply and demand is formulated. A one-leader, $\mathrm{N}$-follower Stackelberg game-based DR algorithm is proposed in Reference [21] to capture the interactions between virtual retailer and Responsive Loads (RLs). In addition to the mentioned drawbacks, each level in the proposed leader-follower frameworks [17-21] has also been modelled as a single bus system without considering the constraints of the network. Besides, the financial interactions between these two levels are not modelled precisely as independent entities. Moreover, none of the reviewed literature considers the reconfigurable topology of the distribution systems.

Recently, by introducing Remotely Controlled Switches (RCSs), hourly reconfiguration can be implemented for short-term day-ahead scheduling of distribution systems [22,23]. In Reference [24], by using hybrid PSO approach, the optimal day-ahead scheduling of RCSs and DERs is proposed in order to minimize the cost function including the cost related to the power losses and switching. Likewise, Anand, et al. [25] solves a simultaneous day-ahead scheduling and hourly reconfiguration problem in distribution systems. In the same subject area, a stochastic optimal operational scheduling of reconfigurable multi-MGs in presence of renewable energy resources is presented in Reference [26] to maximize the total profits.

To the best of our knowledge, none of the reviewed literature provides a bi-level optimization scheduling for decision making of DSO and reconfigurable multi-MGs. In the lights of foregoing 
discussions, in this paper the stochastic one-leader multi-follower-type bi-level model is proposed to embody Distribution System Operator-Microgrid Operators (DSO-MGOs) gaming strategy. In this model, for the upper-level optimization problem, the total cost of the system, including the costs related to power losses, switching actions, exchanged power with wholesale market and exchanged power between DSO and MGs is minimized from DSO's point of view. In the lower-level optimization problem, the profit of each MGO is maximized. To this end, the difference between revenues and costs related to DERs, RLs, consumed power by each MG, exchanged power among MGs and exchanged power between DSO and MGs is maximized. The amount of exchanged power between DSO and each MGO is considered as a mutual decision variable which links the two optimization levels to each other. Since the resulting model is a non-linear bi-level optimization problem, it is then transformed into a single-level Mixed-Integer Second-Order Cone Programming (MISOCP) problem through KKT optimality conditions and linearization techniques. To verify that each final solution resulted from the transformed single-level MISOCP problem is a Nash equilibrium, a single-iteration diagonalization technique is implemented.

In brief, the major contributions of this paper are highlighted as follows:

- A one-leader multi-follower-type bi-level model considering ESSs in an uncertain environment is proposed,

- An optimal day-ahead scheduling of multi-MG distribution systems is proposed considering DR and hourly reconfiguration actions,

- Modelling the DSO's decision-making problem which is affected not only by the exchanged power between DSO and each MG as a mutual decision variable but also by the reactions of interconnected MGs in an hourly reconfigurable environment.

The remainder of the paper is organized as follows: In Section 2, the conceptual framework of the proposed DMS is explained. The bi-level formulation of the optimization problem is proposed in Section 3. Case study and simulation results are discussed in Section 4. Finally, Section 5 summarizes the contribution of the paper.

\section{Conceptual Framework of the Proposed DMS}

In this study, it is assumed that MGs have their own private owners who seek profit maximization, while DSO utilizes the DMS software to minimize the total cost of the distribution system. Thus, DSO and MGs have their own inherently conflicting objectives in optimal scheduling of energy-related generation and consumption units, which can be modelled as a bi-level optimization problem. Figure 1 shows the framework of the proposed bi-level model in the form of a one-leader multi-follower structure for DMS. The leader (which plays at the upper-level in our proposed DMS) is DSO who is responsible for minimizing the total operating costs, whereas the followers (act in the lower-level of the proposed DMS) are MGOs aiming to maximize their own profit. DSO is connected to the upstream utility grid and interacts with networked MGs, while MGOs not only feed the local demand but also exchange energy with each other to make more profit. Thus, the exchanged powers between DSO and multi-MGs are considered as coupling variables between these levels, which enforce decision maker to consider a multi-criteria action plan. It can be seen that DMS receives the network model, retail prices, cost function of DERs, RLs bilateral contracts and forecasted data of load profile, PVs output power and the wholesale market prices as input data. Finally, proposed bi-level DMS returns the optimal amount of exchanged power among MGs, exchanged power between DSO and MGs, Micro Turbines (MTs) and ESSs dispatches, RLs participations, wholesale market purchases and RCSs status during each time interval. Changing the status of RCSs in short-term results in a reconfigurable topology of distribution system, which represents a flexible multi-MG distribution system. 


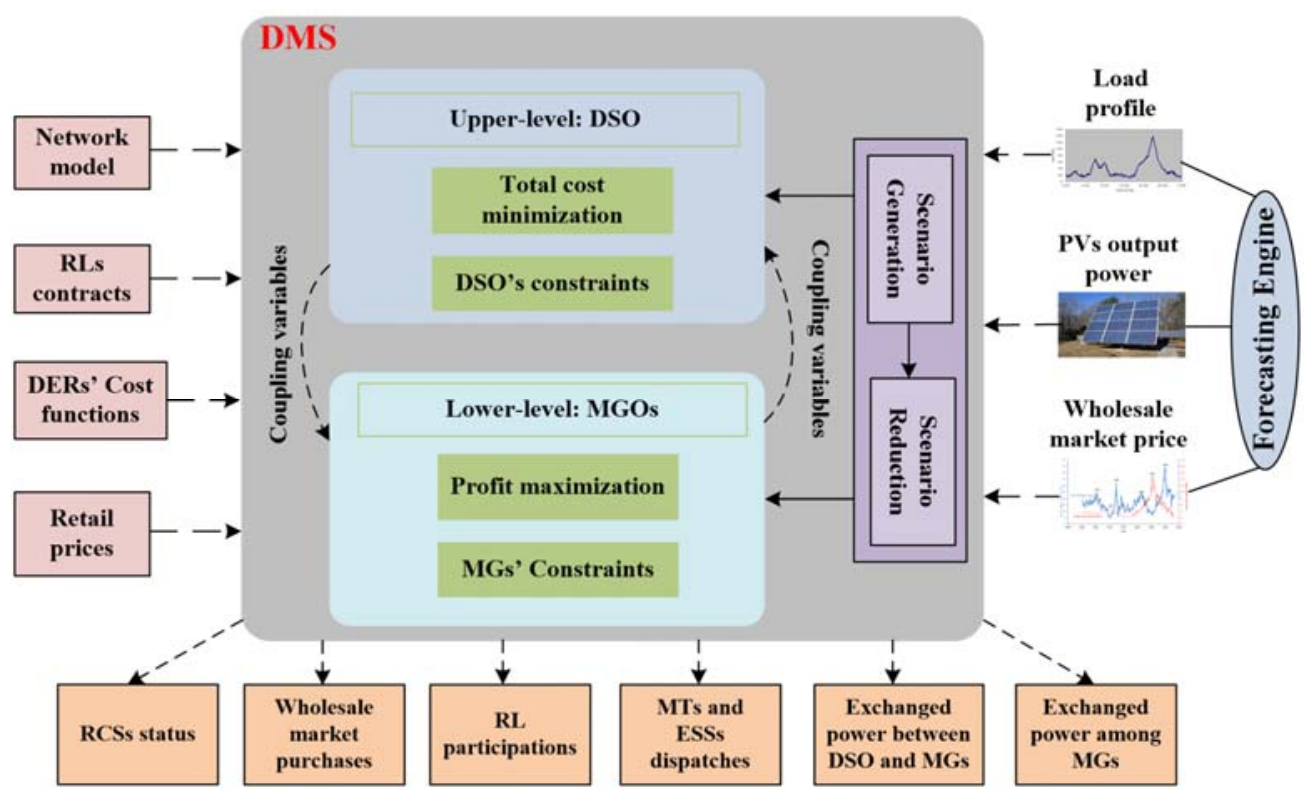

Figure 1. Framework of the proposed bi-level Distribution Management System (DMS).

\section{Uncertainty Modelling}

In the proposed DMS, there are some uncertain parameters such as solar irradiance, wholesale market price and electrical load demand. To study the uncertainty nature of these input data, a stochastic scenario-based method using the mean values (i.e., day-ahead prediction) with different probability density functions is implemented. The scenario generation process is done by Monte-Carlo simulation on predicted mean values while forecasting errors are considered within appropriate probability density functions through associated standard deviations (STDs). The total number of generated scenarios can be calculated by (1) considering constraint (2).

$$
\begin{gathered}
N_{s}=N_{W M} \times N_{P V} \times N_{L} \\
\sum_{s=1}^{N_{s}}\left(\pi_{s}^{W M} \times \pi_{s}^{P V} \times \pi_{s}^{L}\right)=1
\end{gathered}
$$

Due to the computational burden limits of the optimization process, a scenario reduction algorithm based on Kantorovich distance [27] method is also incorporated to decrease the number of generated scenarios into a smaller set representing well enough the uncertainties.

\section{Bi-Level Formulation of the Optimization Problem}

Problem formulation of the bi-level model for operational scheduling of the examined multi-MG distribution system and related solution methodology are described in the following subsections.

\subsection{Upper-Level: DSO}

The objective function of the upper-level is defined as:

$$
O F_{D S O}=\operatorname{Min}\left[\sum_{S_{W M}=1}^{N_{W M}} \pi_{s}^{W M}\left(\sum_{t=1}^{24}\left(C_{t}^{\text {loss }}+C_{t}^{S W}+C_{t}^{W M}+C_{t}^{M G 2 D S O}\right)\right)\right]
$$


As can be seen, the objective function of the upper-level aims at minimizing the total cost from DSO's point of view, which includes different cost terms. The first term denotes the cost of power losses as proposed in (4).

$$
C_{t}^{\text {loss }}=\rho^{\text {loss }} P_{t}^{\text {loss }}
$$

where, the amount of active power losses $P_{t}^{\text {loss }}$ can be calculated by (16). The second term which is represented by (5) denotes the switching cost of RCSs.

$$
C_{t}^{S W}=\rho^{S W} \sum_{s w=1}^{N_{s w}}\left|X_{s w, t, s}-X_{s w, t-1, s}\right|
$$

The absolute value in (5) can be avoided by introducing new auxiliary variables as follows:

$$
\begin{aligned}
& X_{s w, t, s}-X_{s w, t-1, s}=Z_{s w, t} \\
& Z_{s w, t}=Z_{s w, t}^{+}-Z_{s w, t}^{-} \\
& \left|Z_{s w, t}\right|=Z_{s w, t}^{+}+Z_{s w, t}^{-} \\
& Z_{s w, t}^{+}, Z_{s w, t}^{-}=0,1
\end{aligned}
$$

The third term in (3) calculates the cost of purchasing/selling active power from/to day-ahead wholesale market at time $t$.

$$
C_{t}^{W M}=\rho_{t}^{W M}\left(P_{t, s}{ }^{W M 2 D S O}-P_{t, s}{ }^{D S O} 2 W M\right)
$$

Finally, the last term stated in (3) denotes the cost of exchanging power between DSO and MGs represented by (8).

$$
C_{t}^{M G 2 D S O}=\rho_{t}{ }^{W M} \sum_{p=1}^{N_{M G}}\left(P_{t, s}{ }^{M G p 2 D S O}-P_{t, s} D S O 2 M G p\right)
$$

\section{Constraints:}

The upper-level objective function is subjected to the following constraints.

- Distribution power flow equations:

The generic form of the branch flow model deals with the currents and powers flowing through the branches is as follows:

$$
\begin{gathered}
V_{i, t, s}-V_{j, t, s}=Z_{i j} I_{i j, t, s} \quad \forall(i, j) \in b r \\
S_{i j, t, s}=V_{i, t, S_{i j, t, s}^{*}}^{*} \forall(i, j) \in b r \\
\sum_{(i, j) \in b r} S_{i j, t, s}-\sum_{(k, i) \in b r}\left(S_{k i, t, s}-z_{k i}\left|I_{k i, t, s}\right|^{2}\right)+y_{i}^{*}\left|V_{i, t, s}\right|^{2}=S_{i, t, s} \quad \forall i=1,2, \ldots, N_{b u s}
\end{gathered}
$$

where, (9) represents the Ohm's law, (10) defines the branch power flow and (11) states the power balance constraint at each bus. By defining new variables $\ell_{i j, t, s}=\left|I_{i j, t, s}\right|^{2}$ and $v_{i, t, s}=\left|V_{i, t, s}\right|^{2}$ as the squared current and voltage magnitudes, respectively and neglecting the phase angles of currents and voltages, the branch flow model is derived as follows:

$$
\begin{gathered}
P_{i, t, s}=\sum_{(i, j) \in b r} P_{i j, t, s}-\sum_{(k, i) \in b r}\left(P_{k i, t, s}-r_{k i} \ell_{k i, t, s}\right)+g_{i} v_{i, t, s} \quad \forall i=1,2, \ldots, N_{b u s} \\
Q_{i, t, s}=\sum_{(i, j) \in b r} Q_{i j, t, s}-\sum_{(k, i) \in b r}\left(Q_{k i, t, s}-x_{k i} \ell_{k i, t, s}\right)+b_{i} v_{i, t, s} \quad \forall i=1,2, \ldots, N_{b u s} \\
v_{j, t, s}=v_{i, t, s}-2\left(r_{i j} P_{i j, t, s}+x_{i j} Q_{i j, t, s}\right)+\left(r_{i j}^{2}+x_{i j}^{2}\right) \ell_{i j, t, s} \quad \forall(i, j) \in b r
\end{gathered}
$$




$$
\ell_{i j, t, s}=\frac{P_{i j, t, s}^{2}+Q_{i j, t, s}^{2}}{v_{i, t, s}} \forall(i, j) \in b r
$$

The amount of active power losses is calculated as:

$$
P_{t}^{\text {loss }}=\sum_{(i, j) \in b r} r_{i j} \ell_{i j, t}
$$

- Voltage limits constraints:

$$
V_{\min }^{2} \leq v_{i, t, s} \leq V_{\max }^{2}, v_{\text {slack }}=1
$$

- Exchanged power limit with the wholesale market:

$$
P_{t, s}^{W M 2 D S O} \leq P_{\max }^{W M 2 D S O}
$$

- Line current limits:

At any time and working conditions, the loading level of the lines/feeders should be in an allowed range.

$$
\ell_{i j, t, s} \leq I_{\max }^{2} \forall(i, j) \in b r
$$

- Network radiality:

To preserve the radial configuration of the distribution network at each optimization interval, constraints (20)-(23) should be met.

$$
\begin{gathered}
N_{b r, t}=N_{b u s, t}-1 \\
\beta_{i j}+\beta_{j i}=X_{b r} \\
\beta_{0 j}=0, j \in N(0) \\
\sum_{j \in N(i)} \beta_{i j}=1
\end{gathered}
$$

Equation (20) indicates that the resulted configuration at each hour should be without any loop in the equivalent tree graph. In case that bus $j$ is the parent of bus $i\left(\beta_{i j}=1\right)$ or bus $i$ is the parent of bus $j$ $\left(\beta_{j i}=1\right)$, according to $(21)$ the respective branch is in the spanning tree $\left(X_{b r}=1\right)$. In order to avoid islanded buses in the final topology, (22)-(23) state that except the substation bus, all the buses should have one parent.

- Number of switching actions:

$$
\sum_{t=1}^{24}\left|X_{s w, t, s}-X_{s w, t-1, s}\right| \leq N_{\max }^{S W}, \forall s w=1,2, \ldots, \mathrm{N}_{s w}
$$

The above nonlinear constraint can be linearized using auxiliary variables as proposed in (6).

- Exchanged power limit between MGs and DSO:

$$
\begin{aligned}
& P_{\min }^{M G 2 D S O} \leq P_{t, s}^{M G p 2 D S O} \leq P_{\max }^{M G 2 D S O} \\
& P_{\min }^{D S O 2 M G} \leq P_{t, s}^{D S O 2 M G p} \leq P_{\max }^{D S O 2 M G}
\end{aligned}
$$




\subsection{Lower-Level: Multi-MGs}

The objective function of the lower-level part aims to maximize the profit of $p$ th MG considering seven different terms.

$$
\begin{aligned}
O F_{M G p} & =\operatorname{Max}\left(\sum_{S_{P V}=1}^{N_{P V}} \pi_{s}^{P V} \sum_{S_{L}=1}^{N_{L}} \pi_{s}^{L} \sum_{t=1}^{24}\left(\text { Income }_{t, s}^{M G p}-\operatorname{Cost}_{t, s}^{M G p}\right)\right) \\
& =\operatorname{Max}\left(\sum_{S_{P V}}^{N_{P V}} \pi_{s}^{P V} \sum_{S_{L}=1}^{N_{L}} \pi_{s}^{L} \sum_{t=1}^{24}\left(\operatorname{Pr}_{p, t, s}^{L}+\operatorname{Pr}_{t, s}^{M G p 2 D S O}+\operatorname{Pr}_{t, s}^{M G p 2 M G q}+\operatorname{Pr}_{p, t, s}^{M T}+\operatorname{Pr}_{p, t, s}^{P V}+\operatorname{Pr}_{p, t, s}^{E S S}+\operatorname{Pr}_{p, t, s}^{D R}\right)\right)
\end{aligned}
$$

Profit terms of the above objective function are defined as follows:

$$
\begin{aligned}
& \underset{p, t, s}{\stackrel{L}{P r}}=\rho_{t}^{L M} \sum_{i \in \psi_{p}}\left(P_{i, t, s}^{L}\right) \\
& \underset{t, s}{M G p 2 D S O}=\rho_{t}^{L M}\left(P_{t, s}^{M G p 2 D S O}-P_{t, s}^{D S O 2 M G p}\right) \\
& \underset{t, s}{M G p 2 M G q}=\rho_{t}^{L M} \sum_{q=1, q \neq p}^{N_{M G}}\left(P_{t, s}^{M G p 2 M G q}-P_{t, s}^{M G q 2 M G p}\right) \\
& \underset{p, t, s}{M T}=-\sum_{i \in \psi_{p}} \rho_{i}^{M T} P_{i, t, s}^{M T} \\
& \underset{p, t, s}{P V}=-\sum_{i \in \psi_{p}} \rho_{i}^{P V} P_{i, t, s}^{P V} \\
& \underset{p, t, s}{\operatorname{ESS}}=-\sum_{i \in \psi_{p}} \rho_{i}^{E S S}\left(P_{i, t, s}^{d c h}-P_{i, t, s}^{c h}\right) \\
& \underset{p, t, s}{\mathrm{Dr}}=-\sum_{i \in \psi_{p}} \sum_{\xi=1}^{\phi} \rho_{\xi, i, t}^{D R} h_{\xi, i, t, s}
\end{aligned}
$$

\section{Constraints:}

The constraints of the operational scheduling problem at the lower-level are as follows:

- Operation limit of MT:

The operation limits of MTs are formulated in (35)-(41). Constraint (35) guarantees that the output power of the MTs are within the lower and upper limit. The ramp up/down rate constraints of MTs are stated by (36)-(37), while (38)-(41) consider the up/down time limits.

$$
\begin{gathered}
P_{\min }^{M T} \leq P_{i, t, s}^{M T} \leq P_{\max }^{M T} \\
P_{i, t, s}^{M T}-P_{i, t-1, s}^{M T} \leq R_{i}^{u p} \times U_{i, t}^{M T} \\
P_{i, t-1, s}^{M T}-P_{i, t, s}^{M T} \leq R_{i}^{\text {down }} \times U_{i, t-1}^{M T} \\
U_{i, t}^{M T}-U_{i, t-1}^{M T} \leq U_{i, t+U p_{i, k}}^{M T} \\
U_{i, t-1}^{M T}-U_{i, t}^{M T} \leq 1-U_{i, t+D n_{i, k}}^{M T} \\
U p_{i, k}=\left\{\begin{array}{ll}
k & k \leq M U T_{i} \\
0 & k>M U T_{i}
\end{array}\right\}
\end{gathered}
$$




$$
D n_{i, k}=\left\{\begin{array}{ll}
k & k \leq M D T_{i} \\
0 & k>M D T_{i}
\end{array}\right\}
$$

- Exchanged power limit with DSO:

$$
\begin{aligned}
& P_{\min }^{M G 2 D S O} \leq P_{t, s}^{M G p 2 D S O} \leq P_{\max }^{M G 2 D S O} \\
& P_{\min }^{D S O 2 M G} \leq P_{t, s}^{D S O 2 M G p} \leq P_{\max }^{D S O 2 M G}
\end{aligned}
$$

- Exchanged power limit between MGs:

$$
\begin{aligned}
P_{\min }^{M G p 2 M G q} \leq P_{t, s}^{M G p 2 M G q} \leq P_{\max }^{M G p 2 M G q} \\
P_{\min }^{M G 2 M G} \leq P_{t, s}^{M G q 2 M G p} \leq P_{\max }^{M G 2 M G}
\end{aligned}
$$

- $\quad$ Limits on operation of ESSs:

$$
\begin{gathered}
P_{\min }^{E S S} U_{i, t}^{c h} \leq P_{i, t, s}^{c h} \leq P_{\max }^{E S S} U_{i, t}^{c h} \\
P_{\min }^{E S S} U_{i, t}^{d c h} \leq P_{i, t, s}^{d c h} \leq P_{\max }^{E S S} U_{i, t}^{d c h} \\
E_{\min } \leq E_{i, t, s} \leq E_{\max } \\
E_{i, t, s}=E_{i, t-1, s}+\eta_{i}^{c h} P_{i, t, s}^{c h}-\frac{P_{i, t, s}^{d c h}}{\eta_{i}^{d c h}}, \forall t \geq 1 \\
E_{i, t, s}=E_{i}^{\text {initial }}, t=0 \\
U_{i, t}^{c h}+U_{i, t}^{d c h} \leq 1
\end{gathered}
$$

- $\quad$ DR program limits:

In this paper, bid-quantity package offers including the amount of load curtailment and related requested prices are considered as the model of RLs. A typical bid-quantity package for the $i$ th node is proposed in Figure 2 and formulated by (52)-(54).

$$
\begin{gathered}
H_{i, \min } \leq h_{i, \xi} \leq H_{i, \xi}, \xi=1 \\
0 \leq h_{i, \xi} \leq\left(H_{i, \xi+1}-H_{i, \xi}\right), \xi=2,3, \ldots, \phi \\
P_{i}^{D R}=\sum_{\xi=1}^{\phi} h_{i, \xi}
\end{gathered}
$$

where, (52) and (53) state that the amount of accepted load curtailment for DR program in each step $(\xi=1$ and $\xi=2,3, \ldots, \phi$, respectively) should be controlled within an allowable range.

- Power balance for pth MG:

$$
\begin{aligned}
& \sum_{i \in \psi_{p}}\left(P_{i, t, s}^{P V}+P_{i, t, s}^{M T}+P_{i, t, s}^{d c h}-P_{i, t, s}^{c h}+P_{i, t, s}^{D R}\right)+P_{t, s}^{D S O 2 M G p}-P_{t, s}^{M G p 2 D S O} \\
& +\sum_{q=1, q \neq p}^{N_{M G}}\left(P_{t, s}^{M G q 2 M G p}-P_{t, s}^{M G p 2 M G q}\right)=\sum_{i \in \psi_{p}}\left(P_{i, t, s}^{L}+P_{i, t, s}^{l o s s}\right)
\end{aligned}
$$




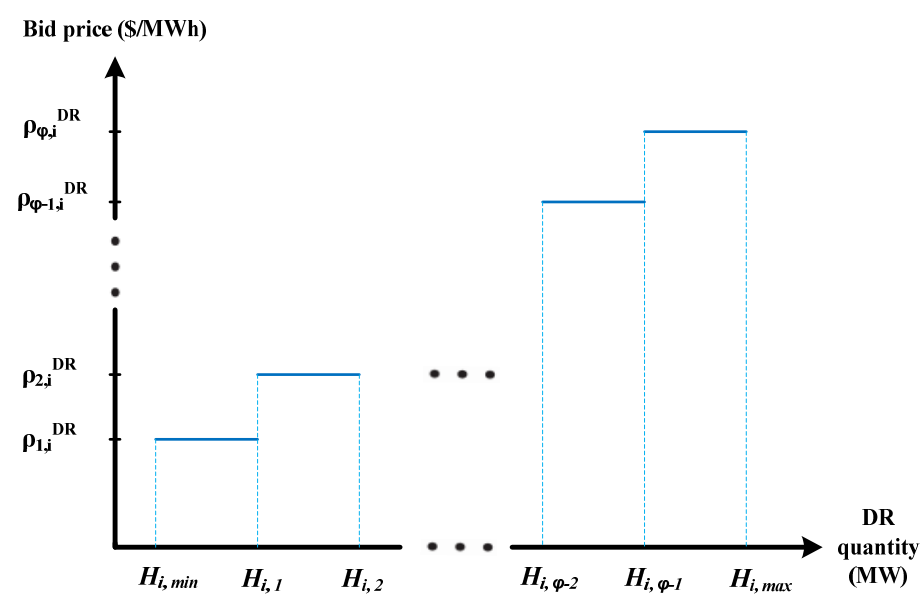

Figure 2. A bid-quantity offer package for $i$ th node.

\subsection{Solution Methodology}

As formulated in previous section, $Y=\left[X_{s w, t} P_{t}^{W M 2 D S O} P_{t}^{D S O 2 W M} P_{t}^{M G \text { 2DSO }} P_{t}^{D S O 2 M G p}\right]$ and $X=\left[P_{t}^{M G p 2 D S O} P_{t}^{D S O 2 M G p} P_{t}^{M G p 2 M G q} P_{t}^{M G q 2 M G p} P_{i, t}^{M T} Q_{i, t}^{M T} P_{i, t}^{c h} P_{i, t}^{d c h} P_{i, t}^{D R}\right]$ are DSO's and MGOs' decision variables, respectively. The amount of exchanged power between DSO and MGs plays an important role in the solution process, which couples the two levels through interactions among players. The proposed bi-level framework cannot be directly implemented in commercial solvers. To resolve this, by adding the KKT conditions of each follower problem, the lower-level problem (27)-(55) is converted into a single-level optimization problem. To solve the resulted single-level MISOCP problem by DSO, DSO has thorough knowledge of the required information pertaining to the MGs. Figure 3 briefly represents the decision-making process outlining the objectives, constraints of the bi-level problem and its transformation into a corresponding single-level MISOCP problem through KKT optimality conditions of the lower-level. The Lagrangian function and related derived KKT conditions of the lower-level problem can be calculated by (A1)-(A36) which are given in Appendix A. In this regard, the collection of non-equality constraints of the lower-level problem are rewritten as greater than or equal to zero constraints. Likewise, all equality constraints of the lower-level problem are rewritten as equal to zero constraints. Indeed, the resulted MISOCP problem is nonlinear because of the complimentary conditions from applying KKTs. To this end, the strong duality theorem is used in Appendix A to linearize the resulted MISOCP problem.

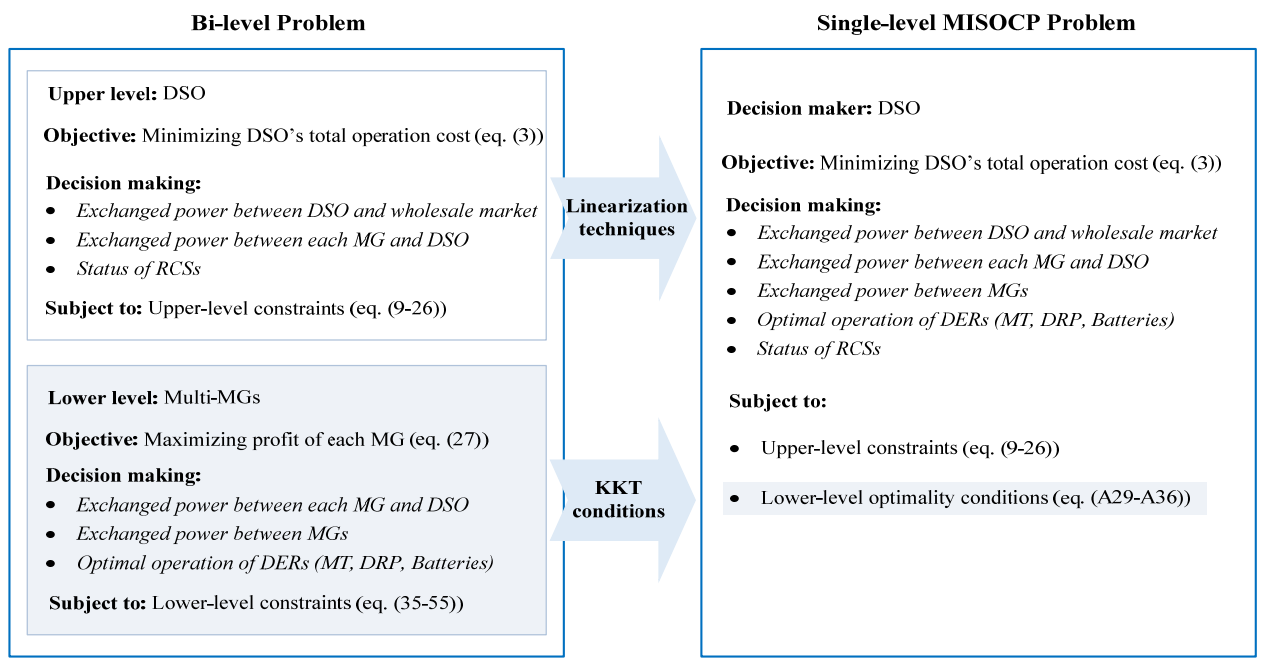

Figure 3. Problem statement and its transformation from a bi-level format into a single-level Mixed-Integer Second-Order Cone Programming (MISOCP) problem. 


\subsection{Verification of Nash Equilibrium}

Generally speaking, a Nash equilibrium of a competitive system indicates that no player has incentive to change its decision. In order to verify that each final solution obtained from the transformed single-level MISOCP problem is a Nash equilibrium, a single-iteration diagonalization technique [28] is used. In this way, offered decision of other players are assumed to be constant which results to a single player problem. To this end, the multi-follower players in the proposed equilibrium problem are divided into $N_{M G}$ (the number of MGs) optimization problems. Optimization problem of each MG is solved with regard to the fixed decision strategies of the rival players (i.e., other MGs). This process is repeated for all of the players. If the decision strategies resulted by diagonalization technique are identical to those obtained by MISOCP problem (i.e., no rival has a profitable unilateral deviation), then the proposed final solution is a Nash equilibrium.

\section{Test and Results}

The bi-level formulation of the proposed model and the solution methodology were discussed in the previous sections. In this section, after introducing the test system, simulation results of the proposed bi-level day-ahead scheduling model are discussed.

\subsection{Case study}

In this paper, the 84-bus $11.4 \mathrm{kV}$ Taiwan power company distribution system [29], as shown in Figure 4, is considered as the test system. This system has three MGs which are interconnected through 13 tie lines. Moreover, it includes 11 feeders and 20 sectionalizing switches. It is assumed that similar type and size of MTs, PVs and ESSs are utilized in MGs with a sitting layout illustrated in Figure 4. All characteristics of DERs and other values for technical parameters are depicted in Table 1 [29]. The predicted values for PV output powers can be extracted from [30]. The support areas of DR programs for the corresponding MGs and their bid-quantity offer packages [31] are provided in Tables 2 and 3, respectively. The forecasted hourly load profile of each MG is shown in Figure 5a, while Figure $5 \mathrm{~b}$ represents the forecasted Real Time Pricing (RTP) of the wholesale market and the retail market prices based on Time-of-Use (TOU) scheme [29].

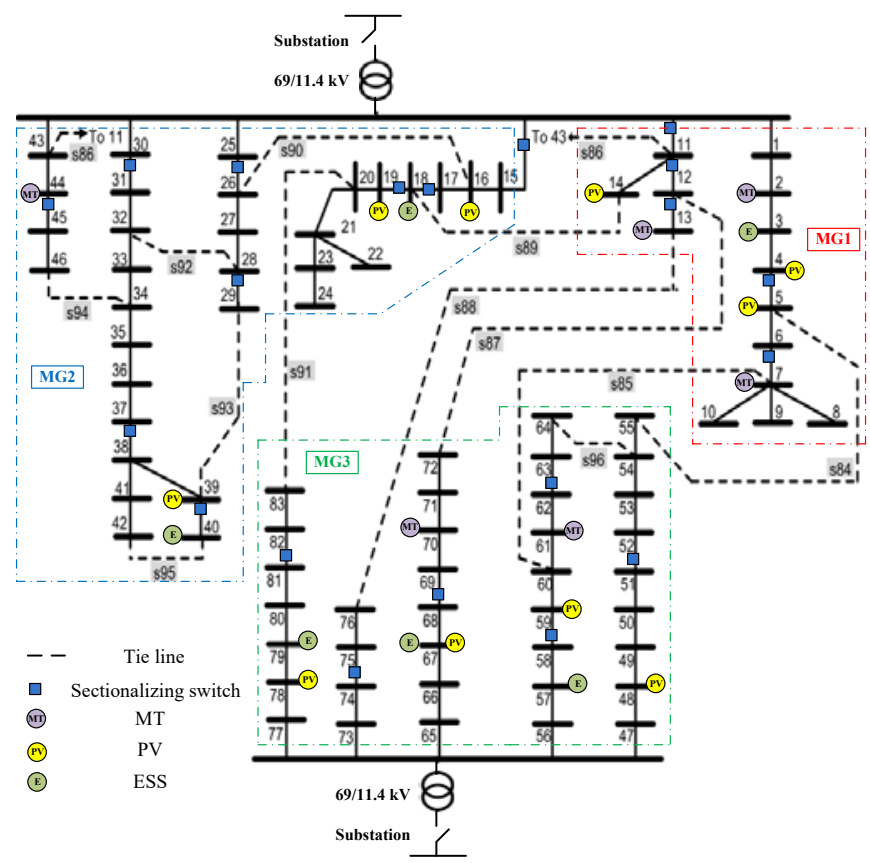

Figure 4. 84-bus Taiwan power company distribution system. 
Table 1. Values for technical parameters [29].

\begin{tabular}{cccc}
\hline Parameter & Value & Parameter & Value \\
\hline$\rho^{\text {loss }}(\$ / M W h)$ & 250 & $\rho_{i}^{M T}(\$ / M W h)$ & 71 \\
$P_{\max }^{W M 2 D S O} / P_{\max }^{D S O M G}(M W)$ & 36 & $\rho_{i}^{P V}(\$ / M W h)$ & 11 \\
$P_{\max }^{M G D S O} / P_{\max }^{D S O 2 M G p}(M W)$ & 20 & $\rho_{i}^{E S}(\$ / M W h)$ & 8 \\
$P_{\max }^{M G G M q} / P_{\max }^{M G q M G p}(M W)$ & 15 & $V_{\min }, V_{\max }(p . u)$. & $0.95,1.05$ \\
$I_{i, j, \max }(k A)$ & 3.8 & $P_{\min }^{M T}, P_{\max }^{M T}(M W)$ & 0,5 \\
$\rho^{S W}(\$)$ & 1 & $N_{\max }^{S W}$ & 8 \\
\hline
\end{tabular}

Table 2. Supported areas of Demand Response (DR) programs in each Microgrid (MG).

\begin{tabular}{cc}
\hline DR program & Buses \\
\hline MG1 & $1,4,6,7,8,12$ \\
MG2 & $16,17,18,22,23,28,31,32,40,45$ \\
MG3 & $49,50,54,59,62,63,67,68,72,80,81,82$ \\
\hline
\end{tabular}

Table 3. Bid quantity offer packages of DR programs [31].

\begin{tabular}{ccccc}
\hline MG No. & \multicolumn{4}{c}{ DR Program [Quantity range } \\
\hline \multirow{2}{*}{ MG1 } & $\mathbf{0 - 0 . 2 ^ { * }}$ & $\mathbf{0 . 2}-\mathbf{0 . 5}$ & $0.5-0.7$ & $0.7-1.1$ \\
& $94^{* *}$ & 97 & 102 & 112 \\
MG2 & $0-0.2$ & $0.2-0.8$ & $0.8-1.4$ & $1.4-1.7$ \\
& 91 & 97 & 104 & 116 \\
\multirow{2}{*}{ MG3 } & $0-0.2$ & $0.2-0.7$ & $0.7-1.3$ & $1.3-1.8$ \\
& 92 & 96 & 102 & 114 \\
\hline
\end{tabular}

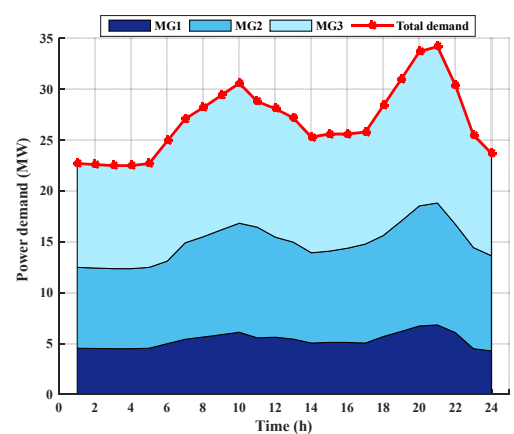

(a)

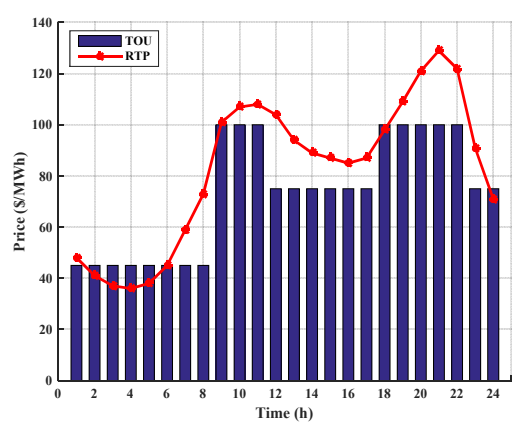

(b)

Figure 5. (a) Power demand; (b) Real Time Pricing (RTP) Wholesale market and Time-of-Use (TOU) retail market prices. 
As proposed in Reference [13], a seven-segment density function for a given normally distributed random variable with mean centred on zero and different STDs can be used to account for generated scenarios. Therefore, the same procedure is adopted for stochastic modelling of the output power of PV units, the wholesale market prices and the electrical load demand. The STD of the PV units' power, the wholesale market prices and the electrical load demand forecast errors are assumed to be $\pm 10 \%, \pm 4 \%$ and $\pm 2 \%$ of their hourly forecasted values, respectively. The Monte-Carlo simulation is performed using MATLAB 2016a and the proposed MISOCP optimization problem is formulated using the General Algebraic Modeling System (GAMS) language and solved by the CPLEX (24.1.2, GAMS Development Corporation, Fairfax, NV, USA) [32] solver.

\subsection{Simulation results}

In order to investigate the satisfactory performance of the proposed model, the problem is addressed in three different case studies. In Case Study 1 (CS1), the interaction of MGs based on the hourly reconfiguration through RCSs is studied, while no interconnection among MGs is considered in Case Study 2 (CS2). In both CS1 and CS2, MGs are equipped with DR programs and ESSs facilities, where MGs in Case Study 3 (CS3) are not possess any of them. In order to deeply evaluate these case studies, three different scenarios are considered in each case. The proposed wholesale market and retail prices in Figure $5 \mathrm{~b}$ are considered as scenario 1, where $10 \%$ increment and decrement in these prices are defined as scenario 2 and scenario 3, respectively. Table 4 summarizes the above CSs and scenarios.

Table 4. Summary of the defined Case studies (CSs) and scenarios.

\begin{tabular}{cccc}
\hline & Scenario 1 & Scenario 2 & Scenario 3 \\
\hline CS1 & Hourly reconfiguration, & Hourly reconfiguration, DR, ESSs, & Hourly reconfiguration, DR, ESSs, \\
CS2 & DR, ESSs, base prices & $10 \%$ increment in prices & $10 \%$ decrement in prices \\
CS3 & DR, ESSs, base prices & DR, ESSs, $10 \%$ increment in prices & DR, ESSs, 10\% decrement in prices \\
\hline
\end{tabular}

The opened RCSs in reconfiguration process of multi-MG distribution system have been tailored in Table 5. By determining the proposed switching sequences at each hour, the optimal topology of the distribution system is resulted. As can be seen in all the final topologies in Table 5 , the network radiality without any islanded buses is preserved, which meet constraints (20)-(23). Moreover, the maximum allowable number of switching actions constraint in the day, stated in (24), is met. Particularly, due to the excess power supply in MG1 and shortage power supply in MG3, RCS no. 84 has the most switching action among all the switches during the day. It is 5 times, which is less than the maximum allowable number of switching actions.

Table 5. Opened Remotely Controlled Switches (RCSs) in reconfiguration process.

\begin{tabular}{cccc}
\hline Hour & Open RCSs & Hour & Open RCSs \\
\hline 1 & $52-85-86-87-88-89-90-91-92-93-94-95-96$ & $12-15$ & $18-26-84-85-86-87-88-91-92-93-94-95-96$ \\
$2-3$ & $52-84-85-86-87-88-89-90-91-92-93-94-95$ & $16-18$ & $84-85-86-87-88-89-90-91-92-93-94-95-96$ \\
$4-8$ & $84-85-86-87-88-89-90-91-92-93-94-95-96$ & $19-21$ & $52-75-85-86-87-89-90-91-92-93-94-95-96$ \\
$9-11$ & $18-52-85-86-87-88-90-91-92-93-94-95-96$ & $22-24$ & $19-59-84-86-87-88-89-90-92-93-94-95-96$ \\
\hline
\end{tabular}

Hourly operation scheduling of the MTs, DR programs and ESSs in CS1 for the defined three scenarios in MG1, MG2 and MG3 are shown in Figures 6-8, respectively. It can be seen that for scenario 2 in all three MGs, due to the increased retail and wholesale market prices, the amount of participated load in DR programs and MTs output power are increased. On the other hand, the amount of responsive loads and MTs output power are decreased in scenario 3. 


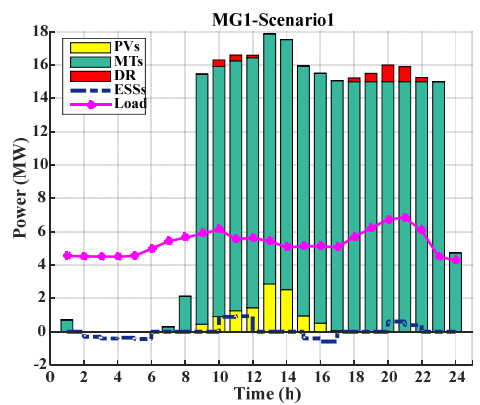

(a)

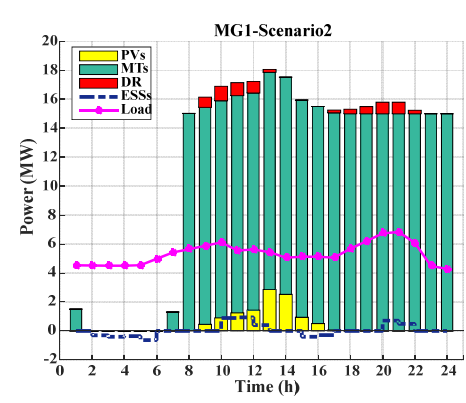

(b)

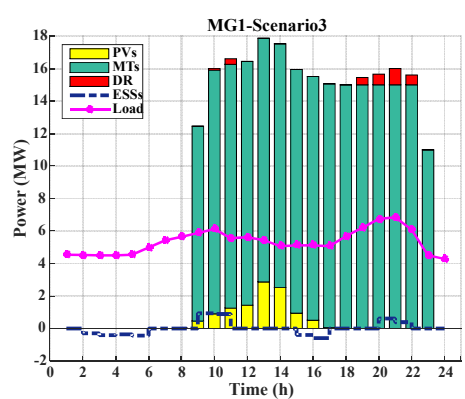

(c)

Figure 6. Hourly operation scheduling of the MTs, DR programs and ESSs in MG1 for CS1: (a) scenario 1 ; (b) scenario 2; (c) scenario 3.

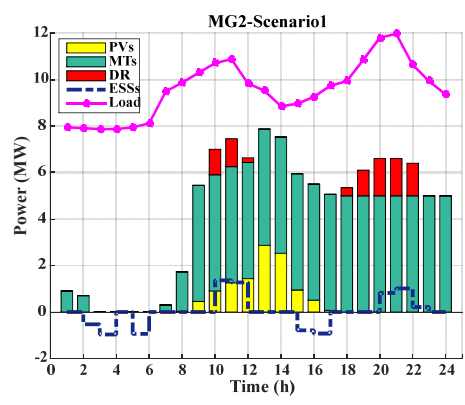

(a)

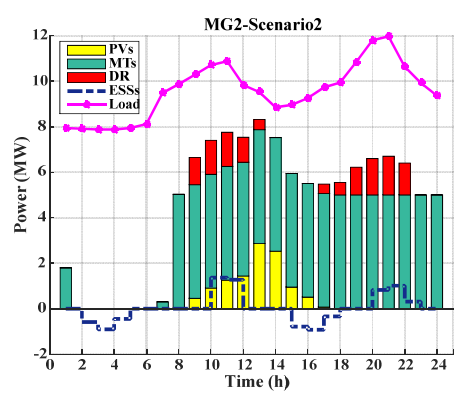

(b)

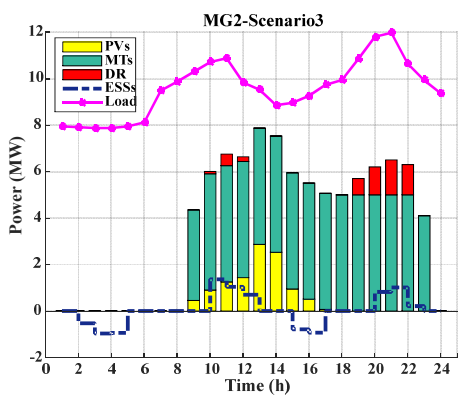

(c)

Figure 7. Hourly operation scheduling of the MTs, DR programs and ESSs in MG2 for CS1: (a) scenario 1 ; (b) scenario 2; (c) scenario 3.

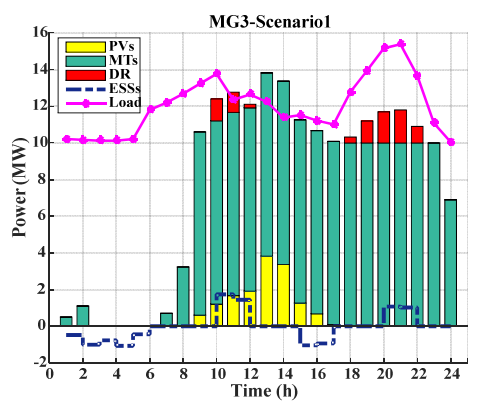

(a)

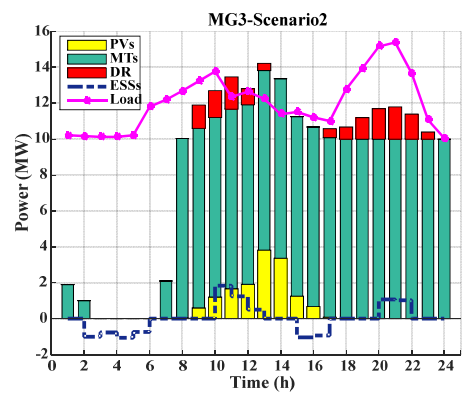

(b)

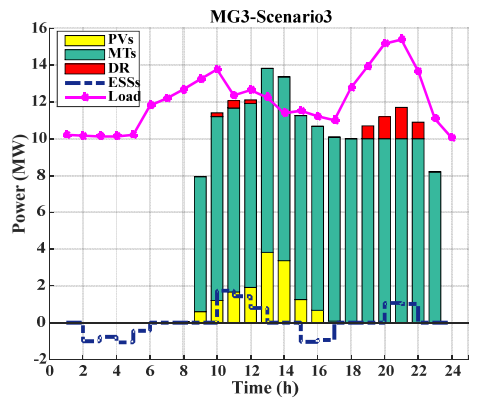

(c)

Figure 8. Hourly operation scheduling of the MTs, DR programs and ESSs in MG3 for CS1: (a) scenario 1 ; (b) scenario 2; (c) scenario 3.

As can be seen in Figures 6-8, curtailable loads are participated in DR programs through the bid-quantity offer packages in peak load periods to flatten the demand curve. In all scenarios, ESSs tend to store power during the hours with low market prices and discharge (i.e., positive values in the figures) the stored power during the high market price periods. Figure 9a, shows the amount of exchanged power among MGs in different scenarios for CS1. Due to the low-load level condition and high penetration of MTs and PVs in MG1, for all scenarios, the power is transmitted from MG1 to MG2 and MG1 to MG3. The negative values represent the reverse power flow (e.g., negative power flow from MG2 to MG3 denotes a flow from MG3 to MG2). Due to the decrement of market prices in scenario 3, MG2 generates less power, thus, the transmitted power from MG1 to MG2 is increased by $6 \%$ compared to scenario 1 . The amounts of exchanged power between DSO and MGs in different scenarios for CS1 to manage the excess or shortage of power supply in each MG are illustrated in Figure $9 \mathrm{~b}$. Based on the lower market prices at time 1:00-8:00 and 24:00, MGOs mainly purchase power from DSO in scenario 1 and scenario 3, while in other hours (i.e., 9:00-23:00), MG1 sells power 
to DSO. In contrast, in scenario 2 at time 8:00 and 24:00, MG1 sells power to DSO because of the higher wholesale market prices. Moreover, due to the lower wholesale market prices in scenario 3 , the total amount of purchased power from DSO is increased by $5 \%$ and $8 \%$, respectively in comparison with those in scenario1 and scenario 2. Figure 10 shows the amounts of exchanged power between each MG and DSO in CS2 for the three defined scenarios. Since no interconnection between MGs is considered in CS2, the power is not exchanged among MGs. This can also be deduced from Figure 10 where due to the higher wholesale market prices in scenario 2, the amount of purchased power from DSO is decreased by $3 \%$ and $5 \%$, respectively. As a whole, in scenario 3, which reflects an operating condition with lower electricity prices, the MGOs prefer to supply more power from the DSO through participating in the market rather than internal energy trading among themselves. Thus, the expensive DERs are switched off in scenario 3 to ensure the economical operation of the distribution system. The amounts of exchanged power between each MG and DSO in CS3 for the three scenarios are shown in Figure 11. As can be seen, the amounts of purchased power from DSO to MG2 and MG3 are increased in peak load periods (i.e., 18:00-23:00) compared to CS2 and CS3. This is because there is no ESSs or DR programs in CS3 to supply power shortage. Figure 12 compares the technical and financial results between CS1, CS2 and CS3 under different scenarios. To get a better insight into this comparative study, the results related to the CS2 scenario 1 are considered as base values and treated as 1.00 per unit (p.u.). As can be seen, in the interconnected topology through the hourly reconfiguration the total loss of the distribution system is less than that of in CS2. Specifically, a reduction of 7\%, $6 \%$ and $8 \%$ in total losses for scenario 1 , scenario 2 and scenario 3 are achieved, respectively, which demonstrates the effect of hourly reconfiguration on loss reduction. Due to the increased power generations from DERs in scenario 2, the total loss in scenario 2 is less than that of scenario 1 and scenario 3 for both CSs. The responsive loads commitment in DR programs in scenario 2 is $27 \%$ and $38 \%$ more than those in scenario 1 and scenario 3, respectively for CS1. On the other hand, consumers' participation in DR actions for CS2 in scenario 2 is $21 \%$ and $29 \%$ more than those in scenario 1 and scenario 3, respectively. Hence, by utilization of hourly reconfiguration for interconnected topology of MGs in CS1, DR activities are increased by $4 \%, 11 \%$ and $2 \%$ in scenario 1, scenario 2 and scenario 3, respectively as compared to CS2. More importantly, the financial profit of MGOs in CS1 are more than those made in CS2 for all scenarios, which demonstrates the efficiency of the proposed interconnected MGs topology. Due to the hourly reconfiguration in CS1, the amounts of profit in scenario 1 are 1.04 p.u., 1.09 p.u. and 1.07 p.u. for MG1, MG2 and MG3, respectively, where these amounts are considered to be 1.00 p.u. for CS2. It is clearly observed from Figure 12 that by applying the hourly reconfiguration through interconnected MGs topology, significant decreases in total cost of distribution system could be achieved in different scenarios. Besides, the amounts of total loss, DR participation, profit of MGs and total costs are presented in Figure 12c. As can be seen, the amounts of losses and total costs are increased compared to CS1 and CS2. On the other hand, the profit of MGs is decreased significantly in different scenarios, which shows the effectiveness of DR programs and ESSs implementation. Through the proposed checking mechanism in Section 3.3, it is verified that the final solutions obtained by the MISOCP problem are Nash equilibrium. For example, for the optimization problem related to the MG1 (MGO2 and MGO3 are considered as fixed decision makers), the profit of MGO1 is 1.04 p.u. and 1 p.u., respectively for CS1 and CS2 in scenario 1, which are consistent with the results shown in Figure 12. 

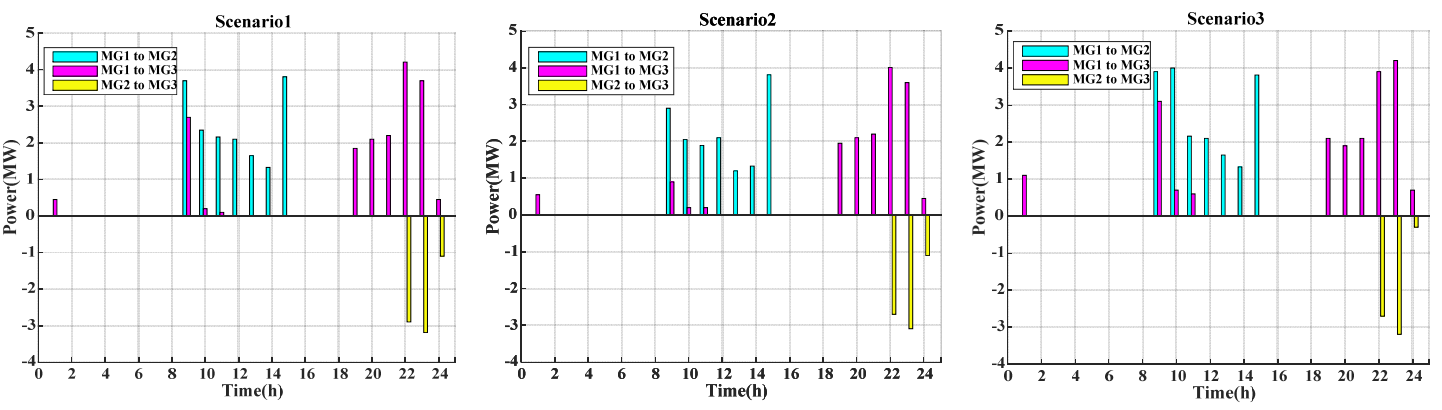

(a)
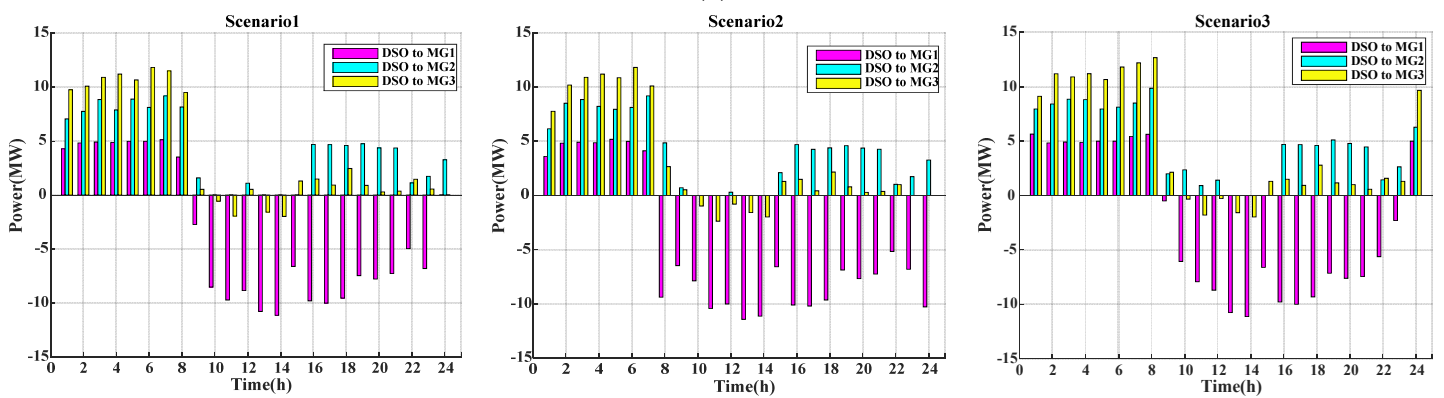

(b)

Figure 9. Exchanged power in different scenarios for CS1: (a) among MGs; (b) between DSO and MGs.
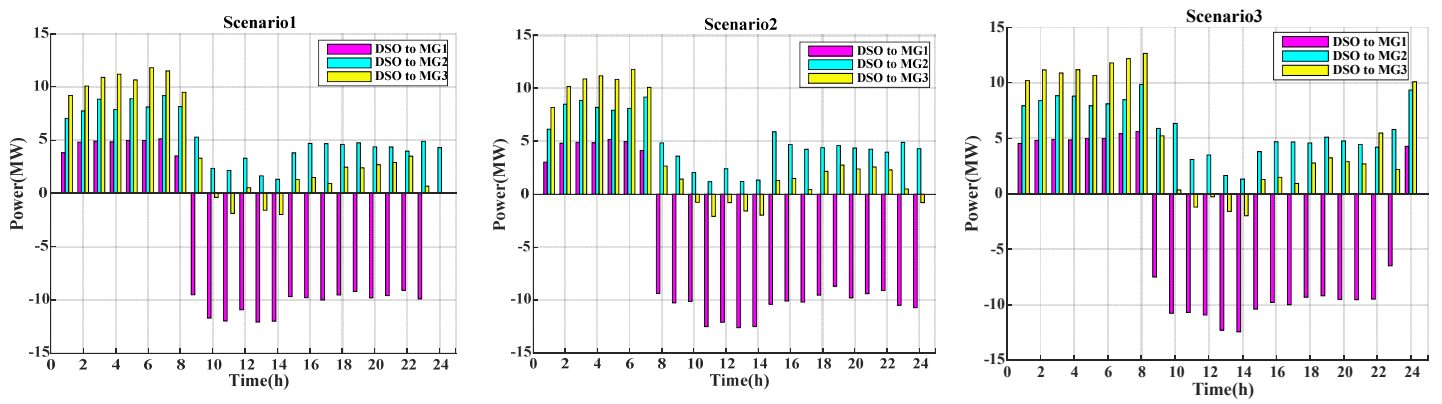

Figure 10. Exchanged power between DSO and MGs in different scenarios for CS2: (a) scenario 1; (b) scenario 2; (c) scenario 3.
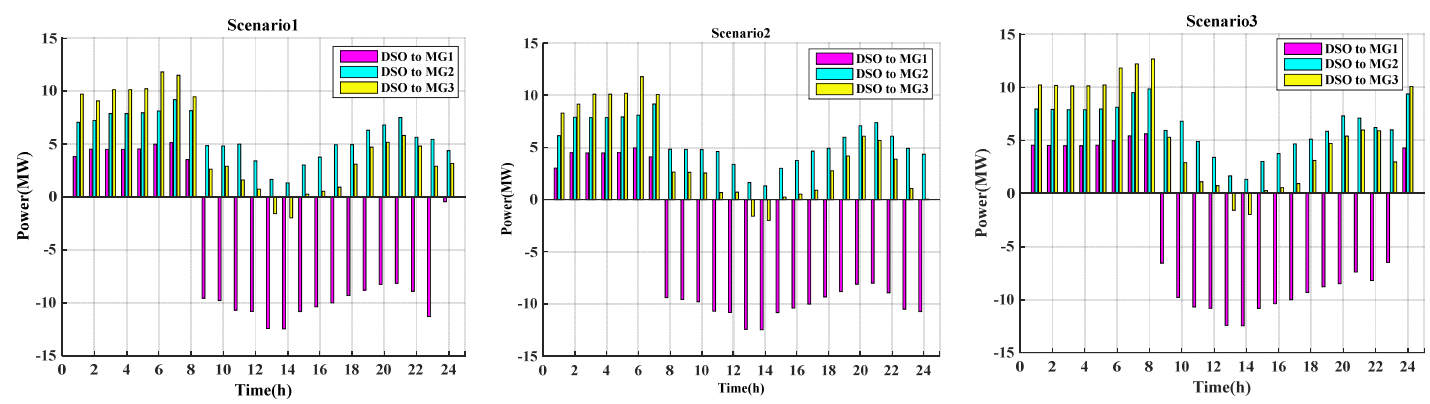

Figure 11. Exchanged power between DSO and MGs in different scenarios for CS3: (a) scenario 1; (b) scenario 2; (c) scenario 3. 


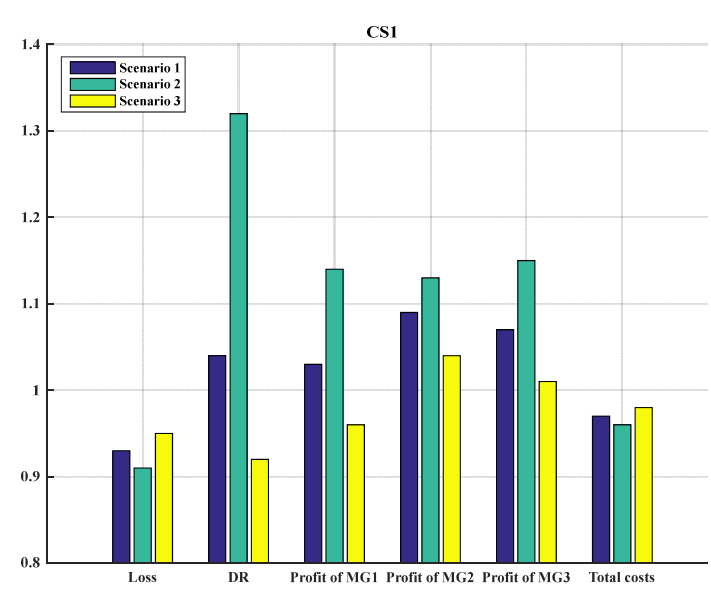

(a)

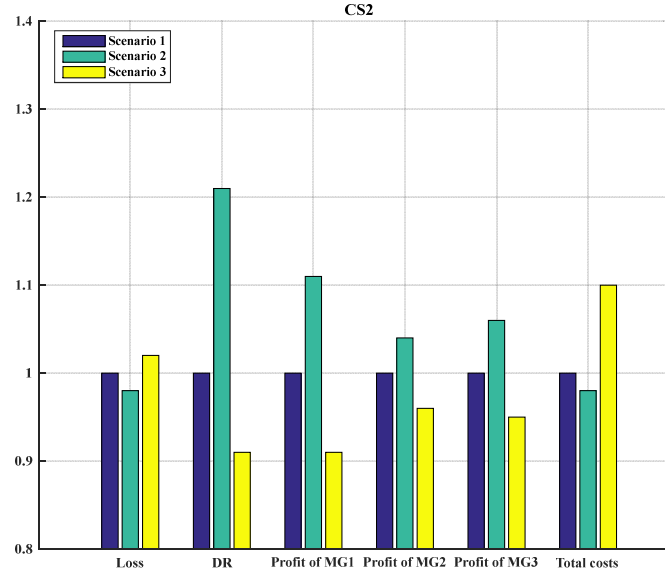

(b)

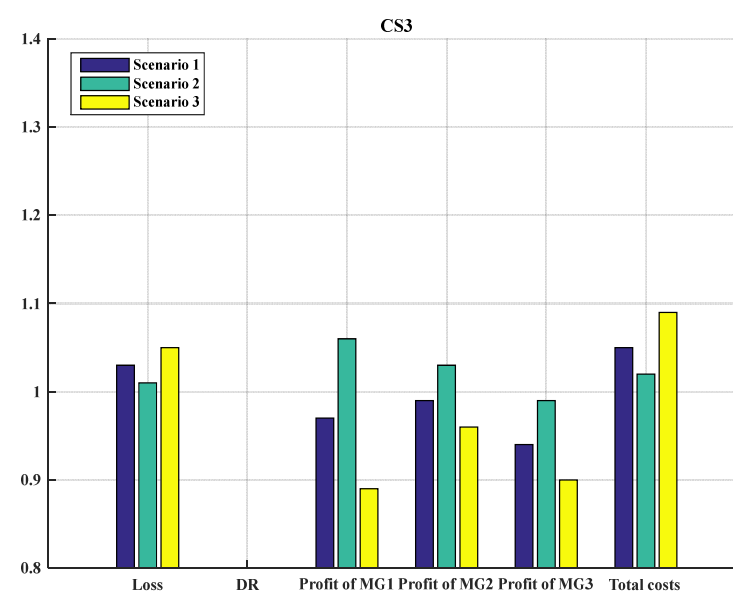

(c)

Figure 12. Comparison of technical and financial results between: (a) CS1; (b) CS2; (c) CS3.

Total load profile of the system after load participation in DR program is shown in Figure 13. It can be seen that RLs are participated in DR programs through the bid-quantity offer packages in peak load periods to flatten the demand curve. Due to the increased retail and wholesale market prices in scenario 2, the amount of participated load in DR programs is increased. Particularly, the peak load has been decreased from 34.2 MW to 29.9 MW, 29.7 MW and 30.7 MW for scenario 1, scenario 2 and scenario 3 , respectively.

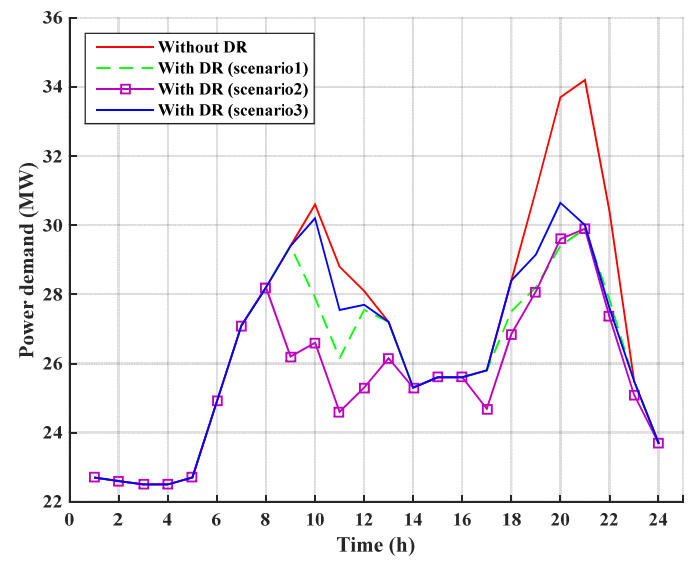

Figure 13. Total load profile with and without considering DR programs in different scenarios. 
From the voltage-security point of view, Figure 14 shows the maximum voltage deviation of busses from 1.00 p.u. for CS1, CS2 and CS3 in three defined scenarios. Due to the higher wholesale market prices and increased power generations from DERs in scenario 2, better bus voltages are achieved. It can be seen that hourly reconfiguration through the interconnected MGs, DR participation and ESSs implementation in CS1 causes better results for bus voltages during the 24h time horizon in all scenarios. Besides, all bus voltages are regulated within their allowable 0.05 p.u. ranges.

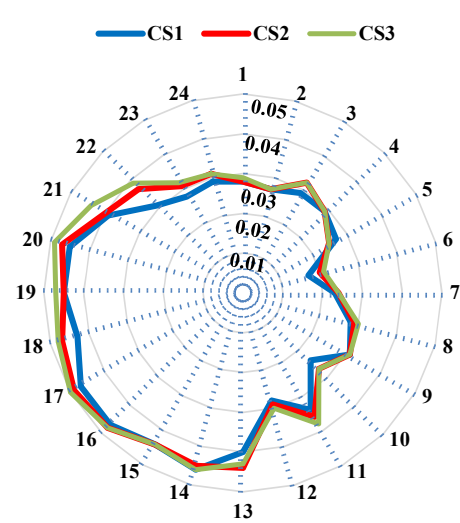

(a)

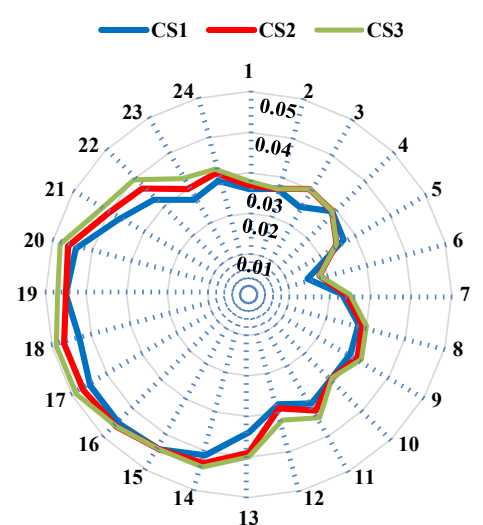

(b)

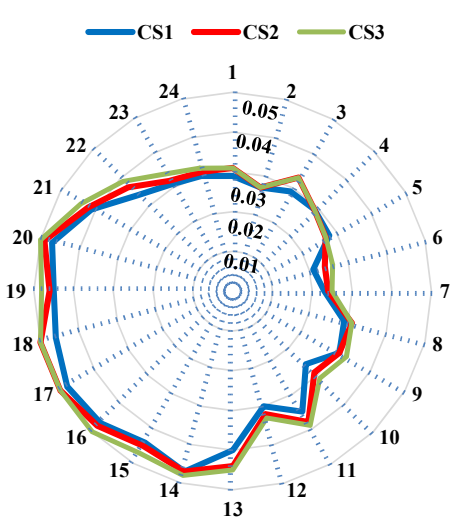

(c)

Figure 14. The maximum voltage deviation of buses for CS1, CS2 and CS3 in: (a) scenario 1; (b) scenario 2 ; (c) scenario 3.

\section{Conclusions}

In this paper, the problem of optimal day-ahead scheduling of reconfigurable multi-MG distribution system considering demand response programs and ESSs in an uncertain environment was investigated. Due to participation of multi-players including DSO and multi-MGs with inherently conflicting objectives in the framework, a one-leader multi-follower-type bi-level optimization model was developed to embody DSO-MGOs gaming strategy. The proposed decision-making model sought to minimize the DSO's total operation cost as the upper-level problem taking into account maximizing the profit of each MG as the lower-level problem. Decision maker was affected not only by the exchanged power between DSO and MGOs as a mutual decision variable but also by the interactions of the interconnected MGs in an hourly reconfigurable topology. KKT optimality conditions and linearization techniques were used to transform the proposed non-linear bi-level optimization problem into a single-level MISOCP problem. The performance of the proposed model was investigated on the 84-bus Taiwan power company distribution system. The simulation results revealed that in conditions where the market prices are relatively higher, MGOs prefer to dispatch first their distributed energy resources including micro turbines, PVs and responsive loads and then exchange power with the interconnected MGs and DSO. However, when the market prices are lower, MGOs prefer to trade energy with DSO. Moreover, it was shown that by implementing hourly reconfiguration through interconnected MGs, the total power losses, the maximum voltage deviation of buses, participation in demand response programs, MGOs' profit and the total system cost could be improved significantly.

Author Contributions: S.E. and A.A.-M. developed the idea and designed the model. The simulation was executed and analysed by S.E. S.E. and A.A.-M. accomplished the writing of the paper. S.J. guided the whole work and reviewed the final paper.

Funding: This research received no external funding.

Conflicts of Interest: The authors declare no conflict of interest. 


\section{Appendix A}

To calculate the KKT conditions of the follower problem, all non-equality constraints of the lower-level problem can be rewritten as greater than or equal to zero constraints proposed in (A1)-(A24). Similarly, the collection of equality constraints of the lower-level are rewritten as equal to zero constraints (A25)-(A28).

$$
\begin{aligned}
& C_{i, p, t, s}^{1}=P_{i, t, s}^{M T}-P_{\min }^{M T} \geq 0: \lambda_{i, p, t, s}^{1}, \forall i, p, t, s \\
& C_{i, p, t, s}^{2}=P_{\max }^{M T}-P_{i, t, s}^{M T} \geq 0: \lambda_{i, p, t, s}^{2}, \forall i, p, t, s \\
& C_{i, p, t, s}^{3}=R_{i}^{u p} \times U_{i, t}^{M T}-P_{i, t, s}^{M T}+P_{i, t-1, s}^{M T} \geq 0: \lambda_{i, p, t, s}^{3}, \forall i, p, t, s \\
& C_{i, p, t, s}^{4}=R_{i}^{\text {down }} \times U_{i, t-1}^{M T}+P_{i, t, s}^{M T}-P_{i, t-1, s}^{M T} \geq 0: \lambda_{i, p, t, s}^{4}, \forall i, p, t, s \\
& C_{i, p, t, s}^{5}=U_{i, t+U p_{i, k}}^{M T}-U_{i, t}^{M T}+U_{i, t-1}^{M T} \geq 0: \lambda_{i, p, t, s}^{4}, \forall i, p, t, s \\
& C_{i, p, t, s}^{6}=1-U_{i, t+D n_{i, k}}^{M T}+U_{i, t}^{M T}-U_{i, t-1}^{M T} \geq 0: \lambda_{i, p, t, s}^{6}, \forall i, p, t, s \\
& C_{i, p, t, s}^{7}=P_{t, s}^{M G p 2 D S O}-P_{\min }^{M G 2 D S O} \geq 0: \lambda_{i, p, t, s}^{7}, \forall i, p, t, s \\
& C_{i, p, t, s}^{8}=P_{\max }^{M G 2 D S O}-P_{t, s}^{M G p 2 D S O} \geq 0: \lambda_{i, p, t, s}^{8}, \forall i, p, t, s \\
& C_{i, p, t, s}^{9}=P_{t, s}^{D S O 2 M G p}-P_{\min }^{D S O 2 M G} \geq 0: \lambda_{i, p, t, s}^{9}, \forall i, p, t, s \\
& C_{i, p, t, s}^{10}=P_{\max }^{D S O 2 M G}-P_{t, s}^{D S O 2 M G p} \geq 0: \lambda_{i, p, t, s}^{10}, \forall i, p, t, s \\
& C_{i, p, t, s}^{11}=P_{t, s}^{M G p 2 M G q}-P_{\min }^{M G 2 M G} \geq 0: \lambda_{i, p, t, s}^{11}, \forall i, p, t, s \\
& C_{i, p, t, s}^{12}=P_{\max }^{M G 2 M G}-P_{t, s}^{M G p 2 M G q} \geq 0: \lambda_{i, p, t, s}^{12}, \forall i, p, t, s \\
& C_{i, p, t, s}^{13}=P_{t, s}^{M G q 2 M G p}-P_{\min }^{M G 2 M G} \geq 0: \lambda_{i, p, t, s}^{13}, \forall i, p, t, s \\
& C_{i, p, t, s}^{14}=P_{\max }^{M G 2 M G}-P_{t, s}^{M G q 2 M G p} \geq 0: \lambda_{i, p, t, s}^{14}, \forall i, p, t, s \\
& C_{i, p, t, s}^{15}=P_{i, t, s}^{c h}-P_{\min }^{E S S} U_{i, t}^{c h} \geq 0: \lambda_{i, p, t, s}^{15}, \forall i, p, t, s \\
& C_{i, p, t, s}^{16}=P_{\max }^{E S S} U_{i, t}^{c h}-P_{i, t, s}^{c h} \geq 0: \lambda_{i, p, t, s}^{16}, \forall i, p, t, s \\
& C_{i, p, t, s}^{17}=P_{i, t, s}^{d c h}-P_{\min }^{E S S} U_{i, t}^{d c h} \geq 0: \lambda_{i, p, t, s}^{17}, \forall i, p, t, s \\
& C_{i, p, t, s}^{18}=P_{\max }^{E S S} U_{i, t}^{d c h}-P_{i, t, s}^{d c h} \geq 0: \lambda_{i, p, t, s}^{18}, \forall i, p, t, s \\
& C_{i, p, t, s}^{19}=E_{i, t, s}-E_{\min } \geq 0: \lambda_{i, p, t, s}^{19}, \forall i, p, t, s \\
& C_{i, p, t, s}^{20}=E_{\max }-E_{i, t, s} \geq 0: \lambda_{i, p, t, s}^{20}, \forall i, p, t, s \\
& C_{i, p, t, s}^{21}=h_{i, \xi}-H_{i, \min } \geq 0: \lambda_{i, p, t, s}^{21}, \forall i, p, t, s, \xi=1 \\
& C_{i, p, t, s}^{22}=H_{i, \xi}-h_{i, \xi} \geq 0: \lambda_{i, p, t, s}^{22}, \forall i, p, t, s, \xi=1 \\
& C_{i, p, t}^{23}=h_{i, \xi} \geq 0: \lambda_{i, p, t}^{23}, \forall i, p, t, \xi=2,3, \ldots, \phi \\
& C_{i, p, t}^{24}=H_{i, \xi+1}-H_{i, \xi}-h_{i, \xi} \geq 0: \lambda_{i, p, t}^{24}, \forall i, p, t, \xi=2,3, \ldots, \phi
\end{aligned}
$$




$$
\begin{gathered}
\sum_{i \in M G p}\left(P_{i, t, s}^{P V}+P_{i, t, s}^{M T}+P_{i, t, s}^{d c h}-P_{i, t, s}^{c h}+P_{i, t, s}^{D R}\right)+P_{t, s}^{D S O 2 M G p}-P_{t, s}^{M G p 2 D S O} \\
+\sum_{q=1, q \neq p}^{N_{M G}}\left(P_{t, s}^{M G q 2 M G p}-P_{t, s}^{M G p 2 M G q}\right)-\sum_{i \in M G p}\left(P_{i, t, s}^{L}+P_{i, t, s}^{l o s s}\right)=0: \lambda_{i, p, t, s^{\prime}}^{25} \forall i, p, t, s \\
E_{i, t, s}-E_{i, t-1, s}-\eta_{i}^{c h} P_{i, t, s}^{c h}+\frac{P_{i, t, s}^{d c h}}{\eta_{i}^{d c h}}=0: \lambda_{i, p, t, s^{\prime}}^{26}, \forall i, p, s, t>1 \\
E_{i, t, s}-E_{i}^{i n i t i a l}-\eta_{i}^{c h} P_{i, t, s}^{c h}+\frac{P_{i, t, s}^{d c h}}{\eta_{i}^{d c h}}=0: \lambda_{i, p, t, s^{\prime}}^{27}, \forall i, p, s, t=1 \\
P_{i}^{D R}-\sum_{\xi=1}^{\phi} h_{i, \xi}=0: \lambda_{i, p, t^{\prime}}^{28}, \forall i, p, t, \xi
\end{gathered}
$$

The Lagrangian function and related derived KKT optimality conditions of the lower-level problem (27)-(55) considering the rewritten form of non-equality and equality constraints of the lower-level problem (A1)-(A28) can be calculated as follows:

$$
\begin{aligned}
& L^{p}=\sum_{S_{P V}}^{N_{P V}} \pi_{s}^{P V} \sum_{S_{L}=1}^{N_{L}} \pi_{s}^{L} \sum_{t=1}^{24}\left[\rho_{t}^{L M} \sum_{i \in M G p}\left(P_{i, t}^{L}\right)+\rho_{t}^{L M}\left(P_{t, s}^{M G p 2 D S O}-P_{t, s}^{D S O 2 M G p}\right)+\rho_{t}^{L M} \sum_{q=1, q \neq p}^{N_{M G}}\left(P_{t, s}^{M G p 2 M G q}\right.\right. \\
& \left.-P_{t, s}^{M G q 2 M G p}\right)-\sum_{i \in M G p}\left(\rho_{i, t}^{M T} P_{i, t, s}^{M T}\right)-\sum_{i \in M G p}\left(\rho_{i, t}^{P V} P_{i, t, s}^{P V}\right)+\sum_{i \in M G p}\left(\rho_{i, t}^{E S S}\left(P_{i, t, s}^{c h}-P_{i, t, s}^{d c h}\right)\right)-\sum_{i \in M G p} \sum_{\xi=1}^{\phi} \rho_{\xi, i, t}^{D R} h_{\xi, i, t} \\
& -\sum_{z=1}^{24} C_{i, p, t, s}^{z} \lambda_{i, p, t, s}^{z}-\lambda_{i, p, t, s}^{25}\left(\sum_{i \in M G p}\left(P_{i, t, s}^{P V}+P_{i, t, s}^{M T}+P_{i, t, s}^{d c h}-P_{i, t, s}^{c h}+P_{i, t, s}^{D R}-P_{i, t, s}^{L}-P_{i, t, s}^{l o s s}\right)+P_{t, s}^{D S O 2 M G p}\right. \\
& \left.-P_{t, s}^{M G p 2 D S O}+\sum_{q=1, q \neq p}^{N_{M G}}\left(P_{t, s}^{M G q 2 M G p}-P_{t, s}^{M G p 2 M G q}\right)\right)-\lambda_{i, p, t, s}^{26}\left(E_{i, t, s}-E_{i, t-1, s}-\eta_{i}^{c h} P_{i, t, s}^{c h}+\frac{P_{i, t s}^{d c h}}{\eta_{i}^{d c h}}\right) \\
& \left.-\lambda_{i, p, t, s}^{27}\left(E_{i, t, s}-E_{i}^{\text {initial }}-\eta_{i}^{\text {ch }} P_{i, t, s}^{c h}+\frac{P_{i, t s}^{d c h}}{\eta_{i}^{d c h}}\right)-\lambda_{i, p, t, s}^{28}\left(P_{i}^{D R}-\sum_{\xi=1}^{\phi} h_{i, \xi}\right)\right] \\
& \frac{\partial L^{p}}{\partial P_{t, s}^{M G 2 M G q}}=\rho_{t}^{L M}\left(N_{M G}-1\right)-\lambda_{i, p, t, s}^{11}+\lambda_{i, p, t, s}^{12}+\lambda_{i, p, t, s}^{25}\left(N_{M G}-1\right)=0 \\
& \frac{\partial L^{p}}{\partial P_{t, s}^{M G q 2 M G p}}=-\rho_{t}^{L M}\left(N_{M G}-1\right)-\lambda_{i, p, t, s}^{13}+\lambda_{i, p, t, s}^{14}-\lambda_{i, p, t, s}^{25}\left(N_{M G}-1\right)=0 \\
& \frac{\partial L^{p}}{\partial P_{i, t, s}^{M T}}=-\sum_{i \in M G p} \rho_{i, t}^{M T}-\lambda_{i, p, t, s}^{1}+\lambda_{i, p, t, s}^{2}+\lambda_{i, p, t, s}^{3}-\lambda_{i, p, t, s}^{4}-\lambda_{i, p, t, s}^{25} \alpha_{t}^{M G p}=0 \\
& \frac{\partial L^{p}}{\partial Q_{i, t, s}^{M T}}=-\sum_{i \in M G p} K_{q}^{M T} \rho_{i, t}^{M T}=0 \\
& \frac{\partial L^{p}}{\partial P_{i, t, \mu s}^{c h}}=\sum_{i \in M G p} \rho_{i, t, s}^{E S S}-\lambda_{i, p, t, s}^{15}+\lambda_{i, p, t, s}^{16}+\lambda_{i, p, t, s}^{25} \alpha_{t}^{M G p}+\left.\lambda_{i, p, t, s}^{26}\right|_{t>1}\left(\eta_{i}^{c h}\right)+\left.\lambda_{i, p, t, s}^{27}\right|_{t=1}\left(\eta_{i}^{c h}\right)=0 \\
& \frac{\partial L^{p}}{\partial P_{i, t, s}^{d c h}}=-\sum_{i \in M G p} \rho_{i, t, s}^{E S S}-\lambda_{i, p, t, s}^{17}+\lambda_{i, p, t, s}^{18}-\lambda_{i, p, t, s}^{25} \alpha_{t}^{M G p}-\left.\lambda_{i, p, t, s}^{26}\right|_{t>1}\left(\frac{1}{\eta_{i}^{d c h}}\right)-\left.\lambda_{i, p, t, s}^{27}\right|_{t=1}\left(\frac{1}{\eta_{i}^{d c h}}\right)=0 \\
& \frac{\partial L^{p}}{\partial P_{i, t, s}^{D R}}=-\sum_{i \in M G p} \sum_{\xi=1}^{\phi} \rho_{\xi, i}^{D R}-\lambda_{i, p, t, s}^{25} \alpha_{t}^{M G p}-\lambda_{i, p, t}^{28}=0
\end{aligned}
$$

Primal feasibility:

Equations (A1)-(A28) state the primal feasibility conditions.

Dual feasibility: 
As all non-equality constraints of the lower-level problem are rewritten as greater than or equal to zero constraints in (A1)-(A24), the respective Lagrangian multipliers (i.e., dual variables) are in the same form. Similarly, as the collection of equality constraints of the lower-level are rewritten as equal to zero constraints in (A25)-(A28), the related dual variables are unrestricted as follows:

$$
\begin{gathered}
\lambda_{i, p, t, s}^{z} \geq 0, \forall z=1,2, \ldots, 24 \forall i, p, t, s \\
\lambda_{i, p, t, s}^{z} \text { Unrestricted, } \forall z=24,25, \ldots, 28 \forall i, p, t, s
\end{gathered}
$$

Complementary slackness conditions:

$$
C_{i, p, t, s}^{z} \lambda_{i, p, t, s}^{z}=0, \forall z=1,2, \ldots, 24 \forall i, p, t, s
$$

The nonlinear constraints in (A39) are transformed into a linear form by big-M method as follows:

$$
\begin{gathered}
C_{i, p, t, s}^{z} \leq M U_{i, p, t, s^{\prime}}^{z}, \forall z=1,2, \ldots, 24 \forall i, p, t, s \\
\lambda_{i, p, t, s}^{z} \leq M\left(1-U_{i, p, t, s}^{z}\right), \forall z=1,2, \ldots, 24 \forall i, p, t, s
\end{gathered}
$$

where, $M$ should be considered as a large positive number.

\section{Nomenclature}

Parameters

$y_{i} \quad$ Admittance between bus $i$ and ground.

$\eta_{i}^{\text {ch }} / \eta_{i}^{\text {dch }} \quad$ Charging/Discharging efficiency of ESS $i$.

$g_{i} / b_{i} \quad$ Conductance/ susceptance of bus $i$.

$\rho_{\mathrm{t}}^{\mathrm{WM}} \quad$ Day-ahead wholesale market electricity price at time $t$ (\$/MWh).

$z_{i j} \quad$ Impedance of branch $i j$.

$E_{i}^{\text {initial }} \quad$ Initial energy storage of ESS $i(\mathrm{MWh})$.

$\rho_{t}^{L M} \quad$ Local market price $(\$ / M W h)$.

$\mathrm{MDT}_{\mathrm{i}} / \mathrm{MUT}_{\mathrm{i}}$ Minimum down/up time for MT $i$.

$N_{W M} / N_{P V} \quad$ Number of generated scenarios for wholesale market price/ PV output power/ demanded load.

$\rho_{\xi \in, t}^{D R} / N_{L} \quad$ Offered price of DR program for load curtailment in step $\xi$ at time $t(\$ / M W h)$.

$\rho_{i}^{E S S} \quad$ Operation \& maintenance cost coefficient of Energy Storage System (ESS) $i$ (\$/MWh).

$\rho_{i}^{M T} \quad$ Operation \& maintenance cost coefficient of $\mathrm{MT} i(\$ / \mathrm{MWh})$.

$\rho_{i}^{P V} \quad$ Operation \& maintenance cost coefficient of PV $i$ (\$/MWh).

$\rho^{\text {loss }} \quad$ Price for power losses $(\$ / \mathrm{MWh})$.

$\rho^{\mathrm{SW}} \quad$ Price of each switching operation (\$).

$\pi_{s}^{W M} / \pi_{s}^{P V}$

$/ \pi_{s}^{L}$

$R_{i}^{u p} / R_{i}^{\text {down }} \quad$ Ramp-up/ramp-down rate of MT $i$.

$r_{i j} / x_{i j} \quad$ Resistance/reactance of branch $i j$.

Variables

$h_{i, \zeta} \quad$ Amount of accepted load curtailment for DR program in step $\xi$ of bus $i$.

$S_{i j, t, s} \quad$ Apparent power of branch $i j$ at time $t$.

$D n_{i, k} / U p_{i, k} \quad$ Auxiliary variables for linear modelling of minimum down/up time limits.

$U_{i, p, t, s}^{z} \quad$ Auxiliary variable used for linearization of the complementary conditions.

$U_{i, t}^{c h} / U_{i, t}^{d c h} \quad$ Binary variable for the charging/discharging status of ESS $i$ at time $t$ (0: when ESS $i$ is not in

the charging or discharging mode).

STD Standard deviation

TOU Time-of-use 
Indices and sets

k Index for On-time/OFF-time limits modelling.

$\mathrm{t} \quad$ Index of time $(t=1,2, \ldots, 24)$.

$\mathrm{i}, \mathrm{j} \quad$ Index of buses $\left(i, j=1,2, \ldots, N_{b u s}\right)$.

br Index of branches $\left(b r=1,2, \ldots, N_{b r}\right)$.

sw Index of RCSs $\left(s w=1,2, \ldots, N_{s w}\right)$.

$\mathrm{p}, \mathrm{q} \quad$ Index of MGs $\left(p, q=1,2, \ldots, N_{M G}\right)$.

$\xi \quad$ Index of steps in bid - quantity packages of DR programs $(\xi=1,2,3, \ldots, \phi)$.

$\psi_{\mathrm{p}} \quad$ Set.

$\mathrm{s} \quad$ Set of scenarios.

$U_{i, t}^{M T} \quad$ Binary variable for the status of MT $i$ at time $t$ (0: when MT $i$ is OFF).

$X_{s w, t, s} \quad$ Binary variable for the status of RCS $s w$ at time $t$ (0: when the related switch is opened)

$C_{t}^{M G 2 D S O} \quad$ Cost of exchanging power between DSO and MGs (\$).

$C_{t}^{W M} \quad$ Cost of exchanging power with wholesale market at time $t(\$)$.

$C_{t}^{S W} \quad$ Cost of switching actions for RCSs at time $t(\$)$.

$I_{i j, t, s} \quad$ Current of branch ij at time $t(\mathrm{kA})$.

$P$ PR $\quad$ Curtailed power of DR for bus $i$ at time $t$ (MW).

$P_{t, s}^{W M 2 D S O} /$

$P_{t, S}^{D S O 2 W M}$

$\lambda_{i, p, t, s}^{z}$

Day-ahead power purchased/sold from/to wholesale market at time $t$ by DSO (MW).

$E_{i, t, s}^{i, p, t, s}$

Dual variable.

$P_{i, t, s}^{c h} / P_{i, t, s}^{d c h}$

$C_{i, p, t, s}^{z}$

Energy storage of ESS $i$ at time $t$ (MWh).

ESS $i$ charge/discharge power at time $t$ (MW).

Greater than or equal to zero constraints.

$L^{p}$

$\alpha_{t}^{M G p}$

$P^{M T}$

$P_{i, t, s}^{M T}$
$P P V$

$Q_{i, t, s}^{P M T}$

$Q_{i, t, s}^{M T}$

$\operatorname{Pr}_{\mathrm{p}, \mathrm{t}, \mathrm{s}}^{\alpha}$

$v_{i, t, s} / \ell_{i j, t, s}$

Lagrangian function of $p$ th lower-level problem related to MG $p$.

Number of buses in the border of MG $p$ at time $t$.

Output active power of MT $i$ at time $t$ (MW).

Output power of PV $i$ at time $t$ (MW).

Output reactive power of MT $i$ at time $t$ (MVAR).

Profit includes loads, PVs, MTs, DR programs and exchanged power between MGs and DSO).

$P_{i, t, s}^{L} \quad$ Total electrical load of bus $i$ at time $t$ (MW).

$P_{t, M G P 2 D S O}^{M}$

$P_{t, S S O 2 M G p}^{D}$ Transmitted power from MG $p$ to DSO/ from DSO to MG $p$ (MW).

$P_{t, s}^{M G p 2 M G q} \quad$ Transmitted power from MG $p$ to MG $q$ at time $t$ (MW).

$V_{i, t, s} \quad$ Voltage magnitude of bus $i$ at time $t$.

\section{References}

1. Kim, M.S.; Haider, R.; Cho, G.J.; Kim, C.H.; Won, C.Y.; Chai, J.S. Comprehensive Review of Islanding Detection Methods for Distributed Generation Systems. Energies 2019, 12, 837. [CrossRef]

2. Holjevac, N.; Capuder, T.; Kuzle, I. Defining key parameters of economic and environmentally efficient residential microgrid operation. Energy Procedia 2017, 105, 999-1008. [CrossRef]

3. Khodaei, A. Provisional microgrid planning. IEEE Trans. Smart Grid 2017, 8, 1096-1104. [CrossRef]

4. Quashie, M.; Marnay, C.; Bouffard, F.; Joós, G. Optimal planning of microgrid power and operating reserve capacity. Appl. Energy 2018, 210, 1229-1236. [CrossRef]

5. Esmaeili, S.; Jadid, S. Economic-Environmental Optimal Management of Smart Residential Micro-Grid Considering CCHP System. Electr. Power Syst. Res. 2019, 1-15. [CrossRef]

6. Uski, S.; Rinne, E.; Sarsama, J. Microgrid as a Cost-Effective Alternative to Rural Network Underground Cabling for Adequate Reliability. Energies 2018, 11, 1978. [CrossRef]

7. Meirinhos, J.L.; Rua, D.E.; Carvalho, L.M.; Madureira, A.G. Multi-temporal Optimal Power Flow for voltage control in MV networks using Distributed Energy Resources. Electr. Power Syst. Res. 2017, 146, $25-32$. [CrossRef] 
8. Gutiérrez-Alcaraz, G.; Galván, E.; González-Cabrera, N.; Javadi, M.S. Renewable energy resources short-term scheduling and dynamic network reconfiguration for sustainable energy consumption. Renew. Sustain. Energy Rev. 2015, 52, 256-264. [CrossRef]

9. Faria, P.; Spínola, J.; Vale, Z. Distributed Energy Resources Scheduling and Aggregation in the Context of Demand Response Programs. Energies 2018, 11, 1987. [CrossRef]

10. Marvasti, A.K.; Fu, Y.; DorMohammadi, S.; Rais-Rohani, M. Optimal operation of active distribution grids: A system of systems framework. IEEE Trans. Smart Grid 2014, 5, 1228-1237. [CrossRef]

11. Lv, T.; Ai, Q.; Zhao, Y. A bi-level multi-objective optimal operation of grid-connected microgrids. Electr. Power Syst. Res. 2016, 131, 60-70. [CrossRef]

12. Feijoo, F.; Das, T.K. Emissions control via carbon policies and microgrid generation: A bilevel model and Pareto analysis. Energy 2015, 90, 1545-1555. [CrossRef]

13. Aghajani, S.; Kalantar, M. Operational scheduling of electric vehicles parking lot integrated with renewable generation based on bilevel programming approach. Energy 2017, 139, 422-432. [CrossRef]

14. Sandgani, M.R.; Sirouspour, S. Energy management in a network of grid-connected microgrids/nanogrids using compromise programming. IEEE Trans. Smart Grid 2018, 9, 2180-2191.

15. Haddadian, H.; Noroozian, R. Multi-microgrids approach for design and operation of future distribution networks based on novel technical indices. Appl. Energy 2017, 185, 650-663. [CrossRef]

16. Gazijahani, F.S.; Ravadanegh, S.N.; Salehi, J. Stochastic multi-objective model for optimal energy exchange optimization of networked microgrids with presence of renewable generation under risk-based strategies. ISA Trans. 2018, 73, 100-111. [CrossRef] [PubMed]

17. Bahramara, S.; Moghaddam, M.P.; Haghifam, M.R. A bi-level optimization model for operation of distribution networks with micro-grids. Int. J. Electr. Power Energy Syst. 2016, 82, 169-178. [CrossRef]

18. Bahramara, S.; Moghaddam, M.P.; Haghifam, M.R. Modelling hierarchical decision making framework for operation of active distribution grids. IET Gener. Transm. Distrib. 2015, 9, 2555-2564. [CrossRef]

19. Jalali, M.; Zare, K.; Seyedi, H. Strategic decision-making of distribution network operator with multi-microgrids considering demand response program. Energy 2017, 141, 1059-1071. [CrossRef]

20. Yu, M.; Hong, S.H. Supply-demand balancing for power management in smart grid: A Stackelberg game approach. Appl. Energy 2016, 164, 702-710. [CrossRef]

21. Yu, M.; Hong, S.H. A Real-Time Demand-Response Algorithm for Smart Grids: A Stackelberg Game Approach. IEEE Trans. Smart Grid 2016, 7, 879-888. [CrossRef]

22. Esmaeili, S.; Anvari-Moghaddam, A.; Jadid, S.; Guerrero, J.M. Optimal Operational Scheduling of Smart Microgrids Considering Hourly Reconfiguration. In Proceedings of the 2018 IEEE 4th Southern Power Electronics Conference (SPEC), Singapore, Singapore, 10-13 December 2018; pp. 1-6.

23. Esmaeili, S.; Anvari-Moghaddam, A.; Jadid, S.; Guerrero, J.M. A Stochastic Model Predictive Control Approach for Joint Operational Scheduling and Hourly Reconfiguration of Distribution Systems. Energies 2018, 11, 1884. [CrossRef]

24. Chen, S.; Hu, W.; Chen, Z. Comprehensive cost minimization in distribution networks using segmented-time feeder reconfiguration and reactive power control of distributed generators. IEEE Trans. Power Syst. 2016, 31, 983-993. [CrossRef]

25. Anand, M.P.; Golshannavaz, S.; Ongsakul, W.; Rajapakse, A. Incorporating short-term topological variations in optimal energy management of MGs considering ancillary services by electric vehicles. Energy 2016, 112, 241-253. [CrossRef]

26. Hemmati, M.; Mohammadi-Ivatloo, B.; Ghasemzadeh, S.; Reihani, E. Risk-based optimal scheduling of reconfigurable smart renewable energy based microgrids. Int. J. Electr. Power Energy Syst. 2018, 101, 415-428. [CrossRef]

27. Gazijahani, F.S.; Salehi, J. Optimal bilevel model for stochastic risk-based planning of microgrids under uncertainty. IEEE Trans. Ind. Inform. 2018, 14, 3054-3064. [CrossRef]

28. Shahmohammadi, A.; Sioshansi, R.; Conejo, A.J.; Afsharnia, S. Market equilibria and interactions between strategic generation, wind, and storage. Appl. Energy 2018, 220, 876-892. [CrossRef]

29. Rostami, M.A.; Kavousi-Fard, A.; Niknam, T. Expected cost minimization of smart grids with plug-in hybrid electric vehicles using optimal distribution feeder reconfiguration. IEEE Trans. Ind. Inform. 2015, 11, 388-397. [CrossRef] 
30. Rabiee, A.; Sadeghi, M.; Aghaeic, J.; Heidari, A. Optimal operation of microgrids through simultaneous scheduling of electrical vehicles and responsive loads considering wind and PV units uncertainties. Renew. Sustain. Energy Rev. 2016, 57, 721-739. [CrossRef]

31. Esmaeili, S.; Anvari-Moghaddam, A.; Jadid, S.; Guerrero, J.M. Optimal simultaneous day-ahead scheduling and hourly reconfiguration of distribution systems considering responsive loads. Int. J. Electr. Power Energy Syst. 2019, 104, 537-548. [CrossRef]

32. CPLEX Optimization Subroutine Library Guide and Reference; ILOG Inc.: Incline Village, NV, USA, 2008.

(C) 2019 by the authors. Licensee MDPI, Basel, Switzerland. This article is an open access article distributed under the terms and conditions of the Creative Commons Attribution (CC BY) license (http://creativecommons.org/licenses/by/4.0/). 